NASA Technical Memorandum 101946

AIAA-89-0993

\title{
Subharmonic and Fundamental High Amplitude Excitation of an Axisymmetric Jet
}

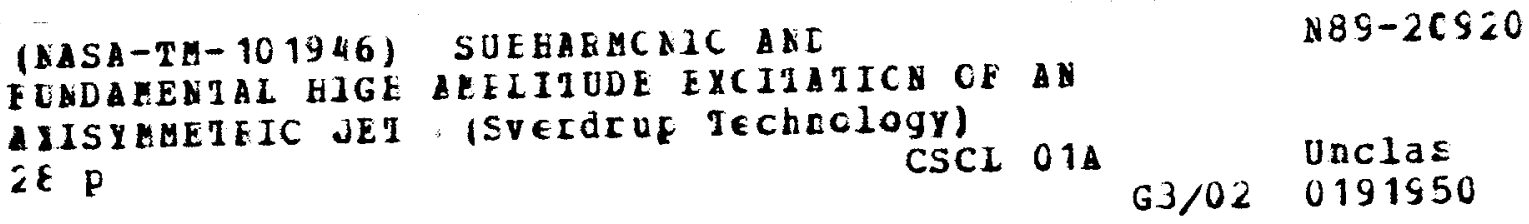

Ganesh Raman

Sverdrup Technology, Inc.

NASA Lewis Research Center Group

Cleveland, Ohio

and

Edward J. Rice

National Aeronautics and Space Administration

Lewis Research Center

Cleveland, Ohio

Prepared for the

2nd Shear Flow Conference

sponsored by the American Institute of Aeronautics and Astronautics

Tempe, Arizona, March 13-16, 1989 


\title{
SUBHARMONIC AND FUNDAMENTAL HIGH AMPLITUDE EXCITATION OF AN AXISYMMETRIC JET
}

\author{
Ganesh Raman \\ Sverdrup Technology, Inc. \\ NASA Lewis Research Center Group \\ Cleveland, Ohio 44135 \\ and \\ Edward J. Rice \\ National Aeronautics and Space Administration \\ Lewis Research Center \\ Cleveland, Ohio 44135
}

SUMMARY

A circular jet was excited simultaneously by two harmonically related tones. This paper reports the results of this excitation on jet behavior for three pairs of Strouhal numbers $\left(S t(D)=f^{*} D / U_{j}=0.2\right.$ and $0.4,0.3$ and 0.6 , 0.4 and 0.8 ). For each case the initial phase difference between the two tones was varied in steps of $45^{\circ}$ for one full cycle, and the amplitude of the fundamental and subharmonic tones were varied independently over the range of 0.1 to 7.0 percent of the jet exit velocity.

Several findings of this study agreed with other published findings, such as a critical amplitude of the fundamental being required for subharmonic augmentation, and the initial phase difference being critical in determining whether the subharmonic is augmented or suppressed. In addition the detailed documentation of several aspects of this phenomenon, all measured in one and the same experimental facility in a controlled manner, brought out several important points that had eluded previous researchers:

(1) At high levels of the fundamental and subharmonic forcing amplitudes, the subharmonic augmentation is independent of the initial phase difference.

(2) Contrary to the earlier belief that "stable pairing" could be produced only with an initial laminar boundary layer, the present work shows that by the two-frequency excitation method, this phenomenon can be induced over a range of conditions for a jet with an initially turbulent boundary layer.

(3) It is seen that two-frequency excitation is indeed more effective than single frequency excitation in jet mixing enhancement. Higher spreading rates seem to go along with higher subharmonic levels.

\section{INTRODUCTION}

The behavior of an axisymmetric shear layer can be controlled in a limited manner by excitation at a single frequency near the preferred mode of the jet. This limitation is due to the fact that the instability wave can enhance mixing only if it continues to grow. When it no longer continues to grow it is said to be "saturated" and its contribution to mixing enhancement ceases. This phenomenon of nonlinearity and saturation has been documented by Crow and Champagne (ref. 1). In a previous paper (ref. 2) results were reported of an 
experiment which looked at the limit of jet mixing enhancement by singlefrequency, plane wave excitation. Saturation was observed in all measured quantities of the jet evolution, i.e., the fundamental wave amplitude, the momentum thickness, the centerline mean velocity and the turbulence intensity. The amplitude of the excitation at which the nonlinearities appeared was 0.3 percent of the jet velocity. The subsequent saturation occurred at an excitation amplitude of around 1 percent of the jet velocity, and at this level the effect on the jet spreading rate was quantified by $U(e x) / U($ une $x)=0.85$, and $\theta(e x) / \theta($ une $x)=1.275$ at $x / D=9$. These experimental results were also compared (in ref. 2) with the predictions of a theoretical model by Mankbadi and Liu (ref. 3).

The degree of jet spreading offered by single frequency plane wave excitation may not seem attractive enough to pursue for practical applications, however when the "preferred mode" frequency becomes neutrally stable, its subharmonic, which is then amplifying at its maximum rate, can be used to cause further mixing enhancement. The development of a subharmonic in a free shear layer has been observed by several researchers. An analysis was presented by Kelly (ref. 4) which showed that there exists a mechanism for the generation of a subharmonic wave in the case of a flow with a hyperbolic tangent velocity profile. It was shown later by Ho and Huang (ref. 5) that the spreading rate of a mixing layer can be manipulated significantly by forcing near the subharmonic of the preferred frequency.

Furthermore, when the shear layer is excited simultaneously by the fundamental and subharmonic, an interaction could occur leading to a large augmentation of the subharmonic amplitude. (In the published literature, depending on the author's investigation technique and perspective, this phenomenon has been referred to as either "resonance" or "pairing".) The axial extent and degree of control over shear layer turbulence and spreading rate is increased if the jet is excited simultaneously by the fundamental and its subharmonic at optimum initial phase difference and amplitudes. Some aspects of this problem have been addressed by Ho and Huang (ref. 5), Durbin and Mckinzie (ref. 6), Arbey and Williams (ref. 7), Zhang et a1. (ref. 8), Mankbadi (ref. 9), Monkewitz (ref. 10), Cohen and Wygnanski (ref. 11), Nikitopoulos and Liu (ref. 12), Ng and Bradley (ref 13) and Hussain and Husain (ref. 14). Arbey and Williams (ref. 7) studied a circular jet simultaneously excited by two different harmonically related acoustic tones. They showed that control can be exercised on the harmonic generation process by varying the phase between the two signals. Zhang et al. (ref. 8) studied a plane mixing layer excited at fundamental and subharmonic frequencies and at various phase differences. The amplification rate of the subharmonic was found to depend strongly on the initial phase difference. A theoretical analysis of the interaction between fundamental and subharmonic instability waves in a turbulent round jet was studied by Mankbadi (ref. 9). The energy integral approach was used to explain the subharmonic augmentation and mean flow manipulation in terms of energy exchanges between the fundamental, subharmonic, fine scale turbulence and the mean flow.

The present work is a parametric study of the effect of simultaneous excitation, at the fundamental and subharmonic frequencies, on the behavior of a circular jet shear layer. The effect of the initial phase difference, Strouhal number pair and amplitudes of the fundamental and subharmonic tones are investigated experimentally. This work incorporates several novel features such as the use of high amplitude excitation devices which can provide a wide range of forcing conditions when used in conjunction with equipment that produce complex 
waveforms. The initial conditions were carefully controlled and monitored in this experiment. The actual phase difference between the two waves was measured in the flow, as opposed to a documentation of the phase difference in the input signal. The above features have helped in producing data that lends new insights into the fundamentals of the two-frequency excitation problem and that will also stimulate future theoretical work. The study also shows the potential for two-frequency excitation to overcome the limitations of single frequency excitation, with regard to mixing enhancement.

\section{EXPERIMENT}

\section{Facility}

The jet excitation facility consisted of a 30-in. diameter settling chamber, an excitation spool-piece, and a convergent nozzle terminated with a 10 in. long straight section having a 3.5-in. exit diameter. Attached to the excitation spool-piece were four Ling electro-pneumatic drivers (Model EPT 9B). Each Ling driver operated at an air supply pressure of $40 \mathrm{lb} / \mathrm{in} .^{2}$. Each driver was capable of producing low frequency ( $f<1000 \mathrm{~Hz}$ ) complex waveforms with an acoustic power up to $4000 \mathrm{~W}$. A photograph and schematic of the jet facility is shown in figure 1.

The 40 psi air that was supplied to the Ling drivers, was exhausted into the plenum tank by elbows which turned the flow upstream into the tank. Downstream of the elbows a screen and honeycomb section was used to condition the flow. The air exhausted by the Ling drivers passed through this flow conditioning and then provided the air flow through the nozzle. Measurements made at the nozzle exit showed that the mean axial velocity profiles were uniformly "top hat" and unaffected by the air supply scheme. The turbulence intensity measured for the unforced jet at the nozzle exit was 1.5 percent as opposed to 0.15 percent in previous experiments (ref. 2) where the Ling drivers were not used. Initially this new system did pose a problem because if the test Mach number was reduced from 0.45 to 0.2 , the pressure to the Ling drivers had to be reduced and this caused a drop in the acoustic output of the drivers, thus changing the forcing levels at the jet exit in a manner that was unacceptable for research. To provide a fine control on the operating Mach number independent of the forcing levels, four bleed valves were installed at the back end of the plenum(not shown in schematic). The test Mach number could thus be reduced from 0.45 to 0.2 by bleeding off the excess air without affecting the acoustic output of the Ling drivers.

A specially fabricated boundary layer trip ring was located 13 in. upstream of the nozzle exit where the diameter of the contracting section was $5.12 \mathrm{in.}$ The trip ring had $83 \mathrm{saw}$ teeth which protrude $4.76 \mathrm{~mm}$ into the flow. The trip ring ensured that the jet exit boundary layer characteristics were invariant with Mach number. The exit boundary layer was turbulent in all tests, similar to a full scale jet exhaust. A polynomial waveform synthesizer generated waveshapes from user supplied mathematical expressions. This signal was amplified by Altec Lansing power amplifiers and fed to the Ling drivers. 
Measurements of mean and fluctuating velocity were made with hot-wire anemometers. The coherent motions were extracted by the phase averaging technique. The phase average is the average of data points having the same phase with respect to a reference signal. The phase averaging rejects the background turbulence and educts the underlying periodic coherent component. The phase average and phase angle difference measurements as well as the spectrum analysis were done using $a$ B \& $K$ dual channel signal analyzer (Model 2025). A computer controlled traversing mechanism was used for probe positioning.

The amplitudes and phase difference input by the waveform synthesizer to the Ling drives are completely different from those measured at the jet exit. The amplifier-driver system and the plenum tank resonance cause significant changes in the phase difference and amplitudes, depending on the frequencies and amplitudes input. Therefore documenting input conditions would be a poor substitute for the actual initial conditions. In the present work the amplitudes of the two waves and the phase difference between them were measured in the flow, at the jet exit, and these constitute a true and accurate representation of the initial conditions.

\section{Initial Conditions}

The experiments were conducted for two jet exit velocities $(M=0.2$ and $M=0.45)$. The turbulence intensity due to the random fluctuations measured at the jet exit centerline was measured to be 1.5 percent of the jet exit velocity. In order to keep the exit boundary layer from playing a parametric role in the experiment, a trip ring was located 10 in. upstream of the jet exit, and it ensured that the exit momentum thickness shape factor, and maximum root mean square fluctuations in the boundary layer remained constant for all test cases. The nozzle exit velocity profile was approximately top hat in shape, and the root mean square profile was uniform in the jet core at the nozzle exit.

The longitudinal velocity spectra measured at the center of the jet exit plane was contaminated by higher harmonics $(3 / 2 f, 2 f)$ from the high amplitude Ling electro-pneumatic driver systems. When traced with downstream distance the $3 / 2 f$ and $2 f$ components were much smaller than the $f$ and the $f / 2$ components and therefore were assumed to not interfere with the excitation process. For example the peak magnitudes of the $f$ and $f / 2$ components were 7 and 20 percent respectively of the jet exit velocity, whereas the $3 / 2 \mathrm{f}$ and $2 \mathrm{f}$ peak magnitudes were 1 and 0.5 percent respectively of the jet exit velocity.

A parametric analysis of the effects of two-frequency excitation is complicated by a multitude of important parameters including the initial phase difference and the absolute and relative forcing amplitudes of the twofrequency components. It is important to note that the initial phase difference $\varphi_{O}$ is defined for velocity and for sine waves.

$$
\tilde{u}=A \sin (2 w t+\varphi O)+B \sin (w t)
$$

In the above equation the initial phase difference is the angle by which the fundamental coherent velocity leads the coherent subharmonic velocity. Three 
pairs of Strouhal numbers were studied. For each case the initial phase difference between the two waves was varied in steps of $45^{\circ}$. The initial forcing level of the fundamental and subharmonic were also varied in the experiment. Table $I(a)$ and (b) shows a summary of the initial conditions for the various two-frequency cases.

\section{DISCUSSION OF RESULTS}

\section{Initial Phase Difference Effect}

Growth of the fundamental and subharmonic on the jet centerline. - The effect of varying the initial phase difference for the Strouhal number pair $0.2,0.4$ is shown in figure 2 . The initial phase difference between the two tones is denoted by $\varphi_{0}$. The fundamental forcing amplitude was 7 percent of the jet exit velocity and the subharmonic amplitude was 0.5 percent. The figure shows that the subharmonic $(f / 2)$ is augmented to very high values for initial phase differences between 225 and $360(0)^{\circ}$ and suppressed for phase differences of 90 and $135^{\circ}$. Phase differences of 45 and $180^{\circ}$ represent the intermediate states. At first glance the fundamental ( $f$ ) appears to be unaffected by the initial phase difference, but a closer scrutiny reveals the kinks in the development of the fundamental which are subtle reminders of the nonlinear interaction between the two waves. Mankbadi, Raman and Rice (ref. 15) explain this dependence theoretically by interpreting the phase difference as being related to the angle between the stresses and the strains of the waves. It was found that the alignment of the stresses and the strains produces augmentation whereas nonalignment produces suppression. Note that the extremely high level of the subharmonic ( 20 percent of the jet velocity) produced by the nonlinear interaction is much higher than can be attained by single frequency excitation.

Many other researchers have also pointed out the importance of the initial phase difference to the subharmonic growth (Monkewitz (ref. 10), Zhang et al. (ref. 8), Mankbadi (ref. 9) and $\mathrm{Ng}$ and Bradley (ref. 13)).

Jet centerline axial velocity. - In the previous section it was shown that depending on the initial phase difference the subharmonic can either be suppressed or augmented. Obviously the result is going to affect the mean velocity and the random turbulence of the jet. The fundamental and subharmonic coherent velocities, the mean velocity and the random turbulence are all coupled together and interact by energy exchanges. Figure $3(a)$ shows the jet centerline velocity plotted versus axial distance for the various phase differences. A steeper descent of the jet centerline velocity signifies a more rapid opening up of the jet plume. There is a very clear dependence between the subharmonic augmentation and the jet centerline velocity. The cases where the subharmonic is augmented to very high levels (fig. 2) correspond to cases where the jet centerline decays most rapidly. This is shown more specifically in figure $3(b)$. In this figure the velocity(excited)/velocity(jet) measured at $x / D=3.5$ is plotted versus the initial phase difference. The unexcited and single frequency ( $S t=0.4$ ) excitation cases are shown for reference. As is readily apparent from the figure, two-frequency excitation is much more effective than single frequency excitation in reducing the jet centerline velocity. 


\section{Effect of Fundamental Forcing Level}

The effect of increasing the forcing level of the fundamental (while keeping the ratio of the fundamental-to-subharmonic levels constant at 15) on the growth of the two waves is shown in figure 4(a) for an initial phase difference of $270^{\circ}$. At low levels of fundamental forcing no subharmonic augmentation is observed, but beyond a critical level of the fundamental the subharmonic augmentation is seen. Monkewitz (ref. 10) has pointed out the existence of this critical level. As seen before, the jet centerline steady velocity is related to the subharmonic augmentation and when the critical level of the fundamental is reached the jet centerline velocity begins to drop, indicating enhanced mixing of the jet ( $f i g .4(b)$ ).

\section{Effect of Strouhal Number Pair}

Figure 5 shows the development of the phase averaged velocities of the fundamental and subharmonic for various initial phase differences. The initial level of the fundamental was equal to that of the subharmonic. Data is shown for three pairs of Strouhal numbers $(0.2,0.4 ; 0.3,0.6 ;$ and $0.4,0.8)$. The rationale for choosing these numbers was the "preferred mode" of the jet, which corresponds to a Strouhal number of 0.3 based on the jet diameter (Crow and Champagne (ref. 1)). In the case of the $0.2,0.4$ pair the two frequencies straddle the "preferred mode" and in the case of $0.3,0.6$, the subharmonic frequency is the preferred mode, whereas in the last case $0.4,0.8$ the subharmonic is slightly higher than the preferred mode case.

The peak amplitude and axial location of the subharmonic in figure $5(b)$ do not depend highly on the initial phase difference. For a fixed initial phase difference the subharmonic peak ("pairing location") depends highly on the Strouhal number pair. The subharmonic peaks at $x / D=2.7$ for the $S t(D)=$ $0.2,0.4$ case ( $f i g .5(a)$ ) and at $x / D=1.2$ for $\operatorname{st}(D)=0.4,0.8$ ( $\mathrm{fig} .5(\mathrm{c})$ ). In the shear layer mode $\left(S t(\theta)=f \theta / U_{j}\right.$ ) the higher frequencies will saturate closer to the jet exit, therefore both the fundamental and the subharmonic peak closer to the jet exit for the $S t(D)=0.4,0.8$ case, whereas the curves peak farthest downstream for the $S t(D)=0.2,0.4$ case. The $S t(D)=0.3,0.6$ case is between these two extremes.

Figure 6 shows the phase difference between the two waves versus axial distance for the various initial phase differences. The initial phase difference for each case has been subtracted out to make the comparison easier. Several comments should be made about the phase difference data in figure 6 . Linear behavior of the excited hydrodynamic modes might be most expected near the nozzle exit where the amplitudes are small and where negligible interaction has taken place. Initial phase difference should not be a factor for linear propagation of instability waves. The phase difference between the fundamental and subharmonic instabilities should change with distance as governed by their wavelength difference and the slight difference in phase velocity. As seen in figure 6 initial phase difference does not have much influence for small $x / D$ especially for the pair, $S t(D)=0.2,0.4$. Unfortunately this result does not have anything to do with hydrodynamic instability propagation for small $x / D$. As clearly seen in figure 2, and less clearly in some parts of figure 5 , the amplitude of the fundamental first drops at low $x / D$ before finally increasing. This represents the fall-off with distance of the acoustic field used for excitation until the rise of the hydrodynamic field dominates at higher $x / D$ 
values. A further complication is that the slope of the phase change is far too great to be explained by the propagation of two spherical waves at the fundamental and subharmonic frequencies. What is being observed at low $x / D$ is the complex nearfield evolution of the propagating and evanescent modes produced by the nozzle exit discontinuity which cannot be further analyzed here. The phase difference at $x / D=0$ is still considered a valid initial condition for comparing the experimental data but it must be realized that hydrodynamic instabilities do not dominate the data until some distance downstream. The influence of initial phase angle on the data in figure 6 is an indicator of nonlinear interactions between the two frequency modes. The spread of the results is maximum at $x / D$ values where subharmonic generation shown in figure 5 is a maximum. Initial phase has the least influence for the pair $S t(D)=0.4,0.8$ where subharmonic augmentation is minimal. This nonlinear two-mode interaction is currently being studied by Mankbadi, Raman and Rice (ref. 15).

Figure 7 shows the jet centerline velocity and the momentum thickness variation with streamwise distance, for the unexcited jet and the three Strouhal number pairs. When the two-frequency excitation is applied, the jet centerline velocity drops and the spreading rate as quantified by the momentum thickness increases. At the high amplitudes used here there is very little difference between the three Strouhal number pairs.

\section{Variation of Subharmonic Forcing Level}

Figure 8 shows the development of the phase averaged velocities of the fundamental and subharmonic for the Strouhal number pair 0.3,0.6. The initial forcing level of the fundamental is kept constant, whereas the forcing level of the subharmonic is varied from 0.1 to 3 percent. In figures $8(a)$ to (c) both the subharmonic peak and the axial location of the peak depend on the initial phase difference. Figure $8(d)$ shows that the peak attained by the subharmonic is not highly dependent on the initial phase difference when the initial subharmonic forcing level is high. The finding that at high forcing amplitudes of the fundamental and subharmonic the subharmonic is always augmented irrespective of the initial phase difference, is not only useful but will have a very favorable impact on the design of practical excitation devices. Figure 9 shows the subharmonic peak versus initial phase difference which is a summary of the cases from figure 8 .

Figure 10 shows the development of the phase difference with axial distance for the four cases of figure 8 , but at phase difference intervals of $90^{\circ}$. As discussed in connection with figure 6 , the initial region of the jet may be dominated by the acoustic near-field which is most evident for high excitation amplitude in figure $10(d)$. The solid lines (phase difference $=0$ ) in figures $10(a)$ and (b) might represent the closest approach to linear behavior since the subharmonic remains at very low levels (see figs. 8(a) and (b) at phase difference $=0$ ). As the initial phase angle and subharmonic excitation level are changed, the slopes of the curves decrease in the range of approximately $1<x / D<3$ (where the subharmonic amplitude is largest) probably due to the nonlinear interaction of the two modes. In this range the phase angle difference is extremely sensitive to initial phase angle especially at high excitation amplitude ( $f$ ig. $10(d)$ ). The steep slope is again attained when the subharmonic amplitude has decayed substantially, which may indicate a change in the nonlinear mechanism or conceivably a return to linearity. 


\section{Jet Mixing Enhancement}

The top row in figure 11 shows the development of the phase averaged velocities along the jet centerline. The four cases represented are: the fundamental alone, the subharmonic alone, both $f$ and $f / 2$ at phase $=180^{\circ}$ and finally both $f$ and $f / 2$ at phase $=0^{\circ}$. Though the waves do not grow to very large amplitudes individually, the two-frequency interaction causes the subharmonic to be augmented to very high levels (or suppressed) depending on the initial phase difference between the two waves. The second row shows the jet centerline velocity along with the unexcited case for comparison. The last row shows the momentum thickness development with axial distance. Very clearly under optimum conditions two-frequency excitation is more effective than single frequency excitation for jet mixing enhancement.

A comparison between all the cases mentioned above is shown in figure 12. The jet centerline velocity and momentum thickness variation are both used as indicators of jet mixing enhancement. A drop in the centerline velocity indicates a higher jet spread for most cases. There is however a reversal in the jet centerline velocity trend between $x / D=1$ and 3 . This is not to be interpreted as a "reversal of mixing". Due to the shortcomings of the jet centerline velocity as a "mixing" indicator the momentum thickness is considered to be a better indicator of jet mixing enhancement. The jet centerline velocity is however retained as it shows a direct relationship between subharmonic augmentation and the "eating up" of the potential core of the jet. The high growth rate of the subharmonic wave causes it to extract energy from the mean flow and this causes the destruction of the potential core. The difference between single and two-frequency optimum excitation results is most pronounced on the jet centerline velocity (fig. 12(a)). In figure 12(b) it is seen that the two-frequency case with phase difference equal to zero produces the same mixing as the single frequency excitation at the fundamental frequency. As seen in figure $11(d)$ (top right) this two-frequency excitation suppresses the coherent subharmonic growth and additional mixing due to forced pairing does not result. Some random pairing may still occur as might be expected in the single frequency excitation case.

Time Traces

Figure 13 shows time traces at various locations on the jet centerline for the (a) unexcited jet, (b) two-frequency case phase $=180^{\circ}$ and (c) twofrequency case phase $=0^{\circ}$. Figure $13(a)$ shows naturally occurring quasiperiodic waves at a period about the same as the subharmonic in figure 13(b). Figure 13(b) shows the forced case at a phase difference of $180^{\circ}$ and indicates that the frequency switches from 440 to $220 \mathrm{~Hz}$ ("pairing") between $x / D=0.5$ and 1 and the subharmonic peaks around $x / D=2$. Further downstream the random turbulence governs the time variations. Figure $13(\mathrm{c})$ shows the forced case at phase $=0$.

At a phase difference of zero the phase averaged measurements indicated a suppression of the subharmonic (fig. 11, top right). The time traces of figure $13(\mathrm{c})$ tell a more realistic story. The merging of vortices (frequency switching) occurs intermittently in space and time. At $x / D=2$ time traces are shown at two different instants, one trace shows a strong velocity component at $220 \mathrm{~Hz}$ and the second trace shows a weak component at $440 \mathrm{~Hz}$. When time averaged and plotted it appears that the subharmonic is suppressed 
(fig. 11, top right), although in reality it is augmented for short periods of time followed by longer periods of suppression. Another point that figure 13(b) makes is the forced pairing that was obtained by the two-frequency forcing in a jet with an initially turbulent boundary layer. Zaman and Hussain (ref. 16) showed that for a forced jet with a laminar exit boundary layer, vortex pairing is regular in space and time, whereas in a jet with a turbulent initial condition, it becomes intermittent in space and time. Figures 5,8 , and $13(b)$ show that under the right conditions stable pairing can be induced by two frequency excitation for a jet with a turbulent initial condition.

$\mathrm{Ng}$ and Bradley (ref. 13) have observed in their flow visualization study that when the jet is forced at more than one frequency the vortex merging process is highly localized and can be "frozen" visually in space by adjusting the phase and frequency of the strobe light, whereas this is not so easily accomplished for the single frequency case. However since they do not document the jet exit boundary layer characteristics for their experiments, no meaningful comparison can be made with the present work.

\section{Radial Distribution of Unsteady Velocities}

Figure 14 shows the radial distribution of unsteady axial velocity which is phase averaged at the fundamental and subharmonic frequencies. Also shown in the figure is the radial distribution of the mean velocity. The measurements are taken at $x / D=2$, where the subharmonic peaks. It is at this location that the vortex pairing processes can be observed (e.g., Moore (ref. 17)). As the amplitude of the subharmonic is much larger than the fundamental, both along the axis and along the radius, it seems that the interaction is only weakly nonlinear and therefore a locally linear parallel flow instability theory will adequately provide the transverse distribution of velocity for both fundamental and subharmonic. Strange and Crighton (ref. 18) have shown this to be true for single frequency cases. Here data on the radial distribution is shown for the two-frequency case. This may justify the shape assumptions used by some researchers in some forms of the energy equation which governs the nonlinear streamwise evolution of the disturbance amplitudes (e.g., Mankbadi (ref. 9)).

\section{CONCLUDING REMARKS}

The effect of exciting an axisymmetric jet simultaneously at fundamental and subharmonic frequencies was parametrically studied. Three Strouhal number pairs were studied $(0.2,0.4 ; 0.3,0.6 ; 0.4,0.8)$. The initial phase difference between the two waves was varied in steps of $45^{\circ}$. The effect of varying the initial forcing levels was also studied. Some of the initial findings concurred with published results. In addition it was found that:

1. At high amplitudes of the fundamental and subharmonic forcing levels the subharmonic augmentation and the axial location of the peak are independent of the initial phase difference. This finding will have a very favorable impact on the design of practical excitation devices.

2. It is seen that two-frequency excitation is indeed more effective than single frequency excitation in jet mixing enhancement. The mixing is quantified by: 
(a) Jet centerline velocity, to show the "eating up" of the potential core

(b) The momentum thickness to show the jet spreading rate

(c) Phase averaged coherent velocities, to indicate the role of the large scale coherent motions in mixing enhancement

3. Contrary to the earlier belief that "stable pairing" could be produced only with initially laminar boundary layers, the present work shows that by using two-frequency excitation this phenomenon can be induced over a range of conditions for a jet with an initially turbulent boundary layer.

\section{REFERENCES}

1. Crow, S.C. and Champagne, F.H., "Orderly Structure in Jet Turbulence," Journal of Fluid Mechanics, Vol. 48, Part 3, Aug. 16, 1981, pp. 547-591.

2. Raman, G., Rice, E.J., and Mankbadi, R.R., "Saturation and the Limit of Jet Mixing Enhancement by Single Frequency Plane Wave Excitation: Experiment and Theory," Ist National Fluid Dynamics Congress, Part 3, AIAA, New York, 1988, pp. 1000-1007 (NASA TM-100882).

3. Mankbadi, R. and Liu, J.T.C., "A Study of the Interactions Between LargeScale Coherent Structures and Fine Grained Turbulence in a Round Jet," Philosophical Transactions of the Royal Society of London, Series A, Vol. 298, No. 1443, Jan. 21, 1981, pp. 541-602.

4. Kelly, R.E., "On the Stability of an Inviscid Shear Layer Which is Periodic in Space and Time," Journal of Fluid Mechanics, Vol. 27, Part 4, Mar. 20, 1967, pp. 657-689.

5. Ho, C.M. and Huang, L.S., "Subharmonics and Vortex Merging in Mixing Layers," Journal of Fluid Mechanics, Vol. 119, June 1982, pp. 443-473.

6. Durbin, P.A. and McKinzie, D.J., "The Effect of Phase Angle on the Evolution of a Frequency and its Subharmonic in a Forced Mixing Layer" (unpublished, private communication).

7. Arbey, H. and Ffowcs Williams, J.E., "Active Cancellation of Pure Tones in an Excited Jet," Journal of Fluid Mechanics, Vol. 149, Dec. 1984, pp. 445-454.

8. Zhang, Y.Q., Ho, C.M., and Monkewitz, P., "The Mixing Layer Forced by Fundamental and Subharmonic," Laminar-Turbulent Transition, V.V. Kozlov, ed., Springer-Verlag, 1985, pp. 385-396.

9. Mankbadi, R.R., "On the Interaction Between Fundamental and Subharmonic Instability Waves in a Turbulent Round Jet," Journal of Fluid Mechanics, Vol. 160, Nov. 1985, pp. 385-419.

10. Monkewitz, P.A., "Subharmonic Resonance, Pairing and Shredding in the Mixing Layer," Journal of Fluid Mechanics, Vol. 188, Mar. 1988, pp. 223-252. 
11. Cohen, J. and Wygnanski, I., "The Evolution of Instabilities in the Axisymmetric Jet. Part 2. The Flow Resulting from the Interaction Between Two Waves," Journal of Fluid Mechanics, Vol. 176, Mar. 1987, pp. 221-235.

12. Nikitopoulos, D.E. and J.T.C. Liu, "Nonlinear Binary Mode Interactions in a Developing Mixing Layer," Journal of Fluid Mechanics, Vol. 179, June 1987, pp. 345-370.

13. Ng, T.T. and Bradley, T.A., "Effect of Multi-Frequency Forcing on the Near Field Development of a Jet," AIAA Paper 87-0054, Jan. 1987.

14. Hussain, A.K.M.F. and Husain, H.S., "Passive and Active Control of Jet Turbulence," Turbulence Management and Relaminarization IUTAM Symposium Bangalore, India 1987. (Springer Verlag Berlin Heidelberg, 1988.)

15. Mankbadi, R.R., Raman, G. and Rice, E.J., "On the Conditions for Resonance Interactions of Instability Waves in the Axisymmetric Jet, "Submitted to Physics of Fluids, 1989.

16. Zaman, K.B.M.Q. and Hussain, A.K.M.F., "Vortex Pairing in a Circular Jet Under Controlled Excitation. Part 1. General Jet Response," Journal of Fluid Mechanics, Vol. 101, Part 3, Dec. 11, 1980, pp. 449-491.

17. Moore, C.J., "The Role of Shear Layer Instability Waves in Jet Exhaust Noise," Journal of Fluid Mechanics, Vol. 80, Part 2, Apr. 25, 1977, pp. $321-367$.

18. Strange, P.J.R. and Crighton, D.G., "Spinning Modes on Axisymmetric Jets, Part I," Journal of Fluid Mechanics, Vol. 134, Sept. 1983, pp. 231-245.

TABLE I. - SUMMARY OF INITIAL CONDITIONS OF TWO-FREQUENCY EXCITATION

(a) $M=0.2$

\begin{tabular}{|c|c|c|c|c|}
\hline $\begin{array}{c}\text { Strouhal } \\
\text { number } \\
\text { pair } \\
\text { St (D) }\end{array}$ & $\begin{array}{l}\text { Fundamental } \\
\text { forcing } \\
\text { ampi tude, } \\
\left(u_{f}\right) / U_{j} \\
\text { (percent) }\end{array}$ & $\begin{array}{l}\text { Subharmonic } \\
\text { forcing } \\
\text { amplitude, } \\
\left(\tilde{u}_{f / 2}\right) / U_{j} \text {, } \\
\text { (percent) }\end{array}$ & $\begin{array}{c}\text { Ratio } \\
\text { fund/sub }\end{array}$ & $\begin{array}{c}\text { Phase } \\
\text { difference, } \\
(\varphi)_{0} \\
\text { deg }\end{array}$ \\
\hline $0.2,0.4$ & 3 & 3 & 1 & 0 to 360 (step 45) \\
\hline $0.3,0.6$ & 3 & $\begin{array}{l}3 \\
0.6 \\
0.2 \\
0.1\end{array}$ & $\begin{array}{r}1 \\
5 \\
15 \\
30\end{array}$ & 0 to 360 (step 45) \\
\hline $0.4,0.8$ & 3 & 3 & 1 & 0 to 360 (step 45$)$ \\
\hline
\end{tabular}

(b) $M=0.45$

\begin{tabular}{|c|c|c|c|c|}
\hline $0.2,0.4$ & 7 & 0.47 & 15 & 0 to 360 (step 45$)$ \\
\hline & $\begin{array}{l}5.7 \\
3.4 \\
2.8 \\
2 \\
1\end{array}$ & $\begin{array}{l}0.38 \\
0.23 \\
0.19 \\
0.13 \\
0.067\end{array}$ & 1 & 270 \\
\hline
\end{tabular}




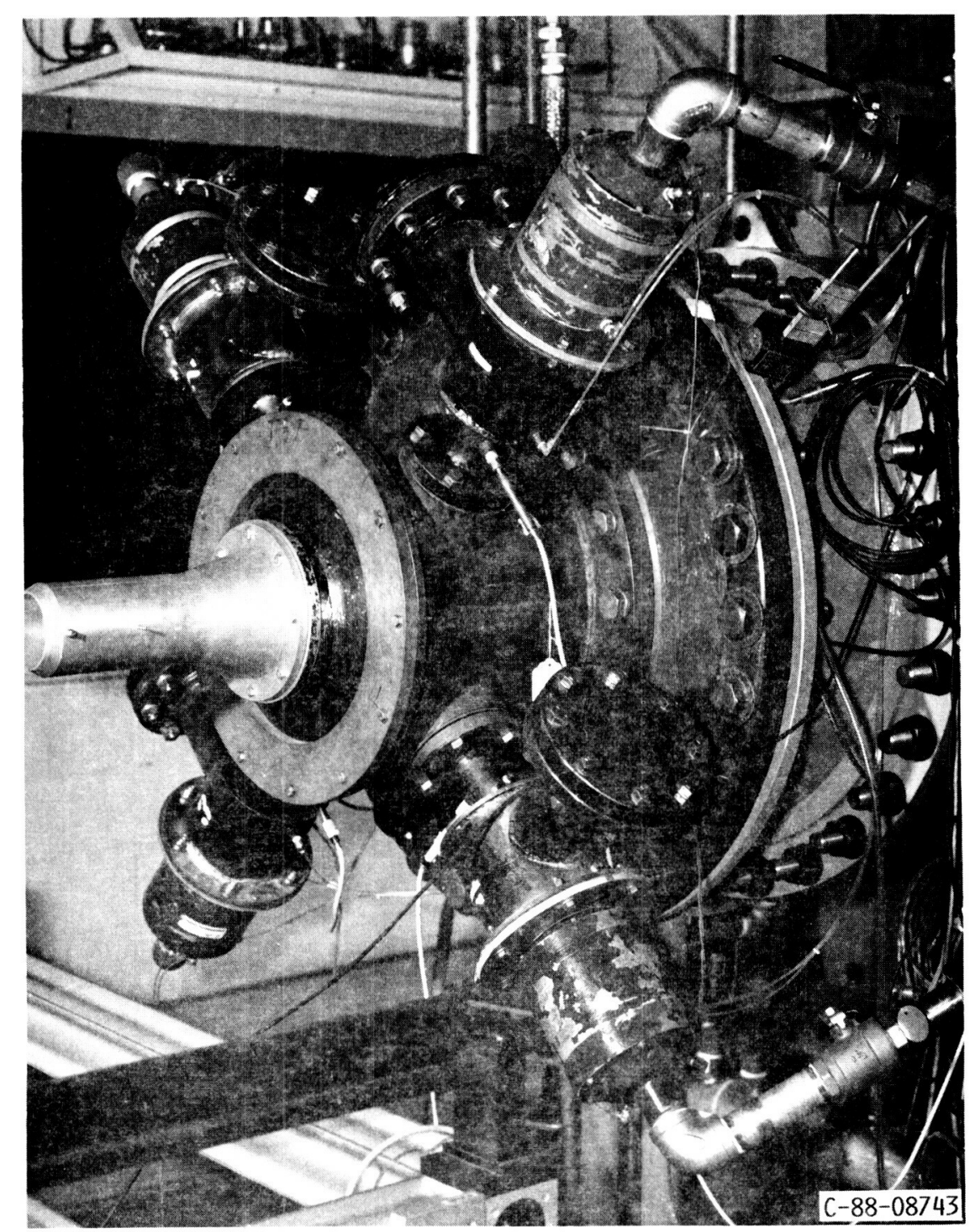

FIGURE 1(a). - AXISYMMETRIC JET HIGH AMPLITUDE EXCITATION RIG. 


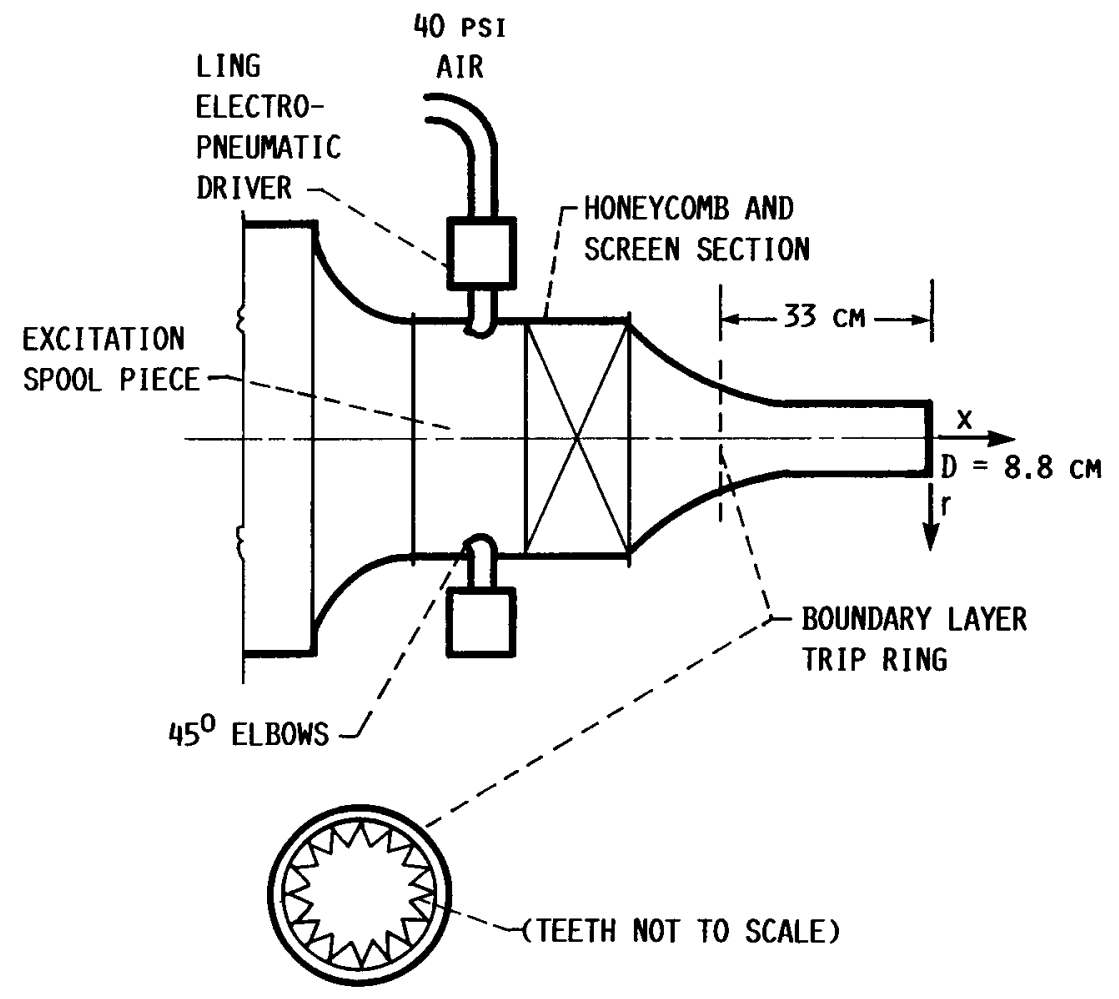

FIGURE $1(b)$. - SCHEMATIC OF HIGH AMPLITUDE EXCITATION FACILITY. 


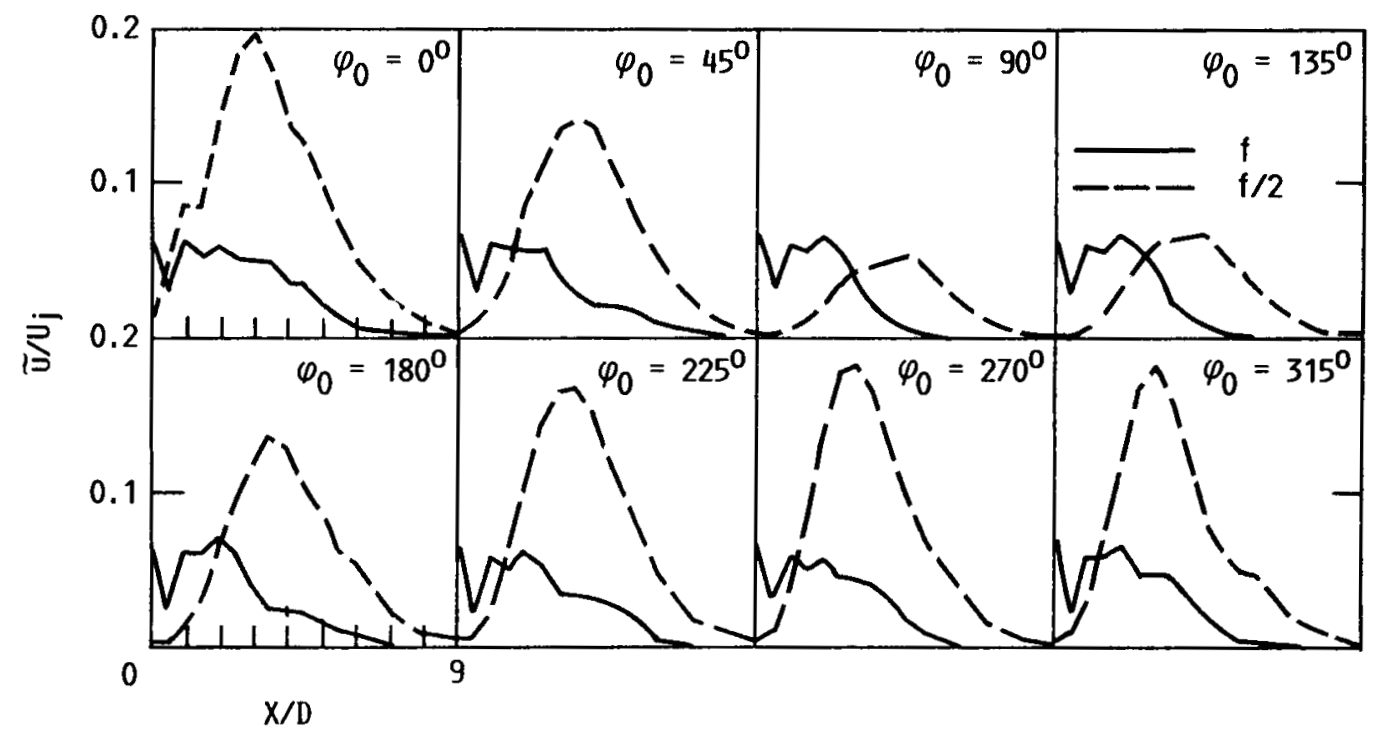

FIGURE 2. - AXIAL EVOLUTION OF PHASE AVERAGE UNSTEADY VELOCITY COMPONENTS ON ON JET CENTERLINE FOR VARIOUS INITIAL PHASE DIFFERENCES. $(S t=0.2,0.4$, $\left.\widetilde{u}_{f_{0}}=0.07 u_{j} \cdot \widetilde{u}_{f / 2}=0.0045 U_{j} \cdot M=0.45.\right)$

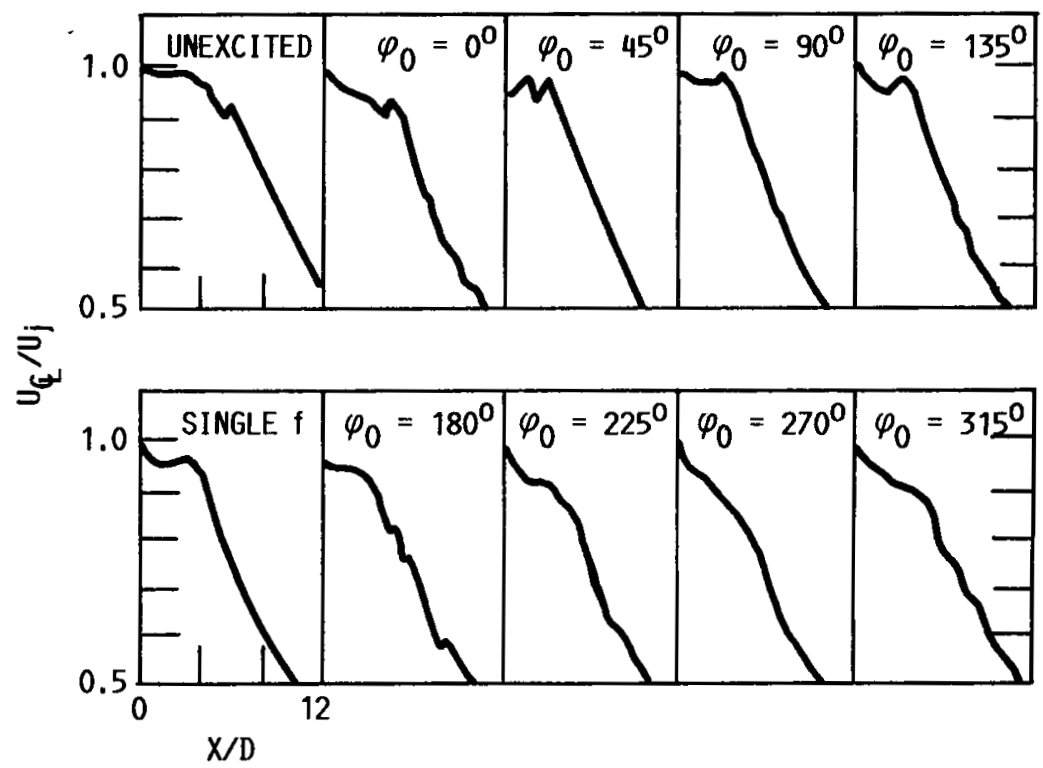

(a) AXIAL VARIATION OF JET CENTERLINE VELOCITY.

FIGURE 3. - TWO-FREQUENCY EXCITATION INFLUENCE ON JET CENTERLINE VELOCITY. $\quad(S t=0.2,0.4, M=0.45$, $\left.\widetilde{u}_{f_{0}}=0.07 U_{j}, \widetilde{u}_{f / 2_{0}}=0.0045 U_{j}.\right)$ 


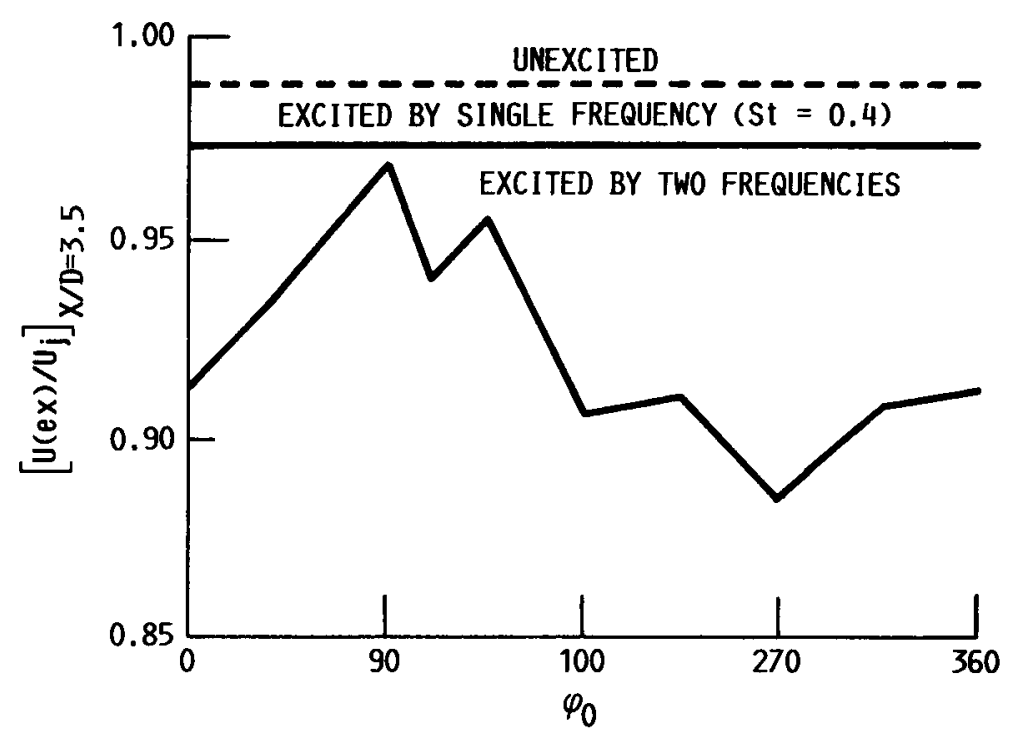

(b) MEAN VELOCITY RATIO (EXCITED/JET EXIT) AT $X / D=3.5$.

FIGURE 3. - CONCLUDED.

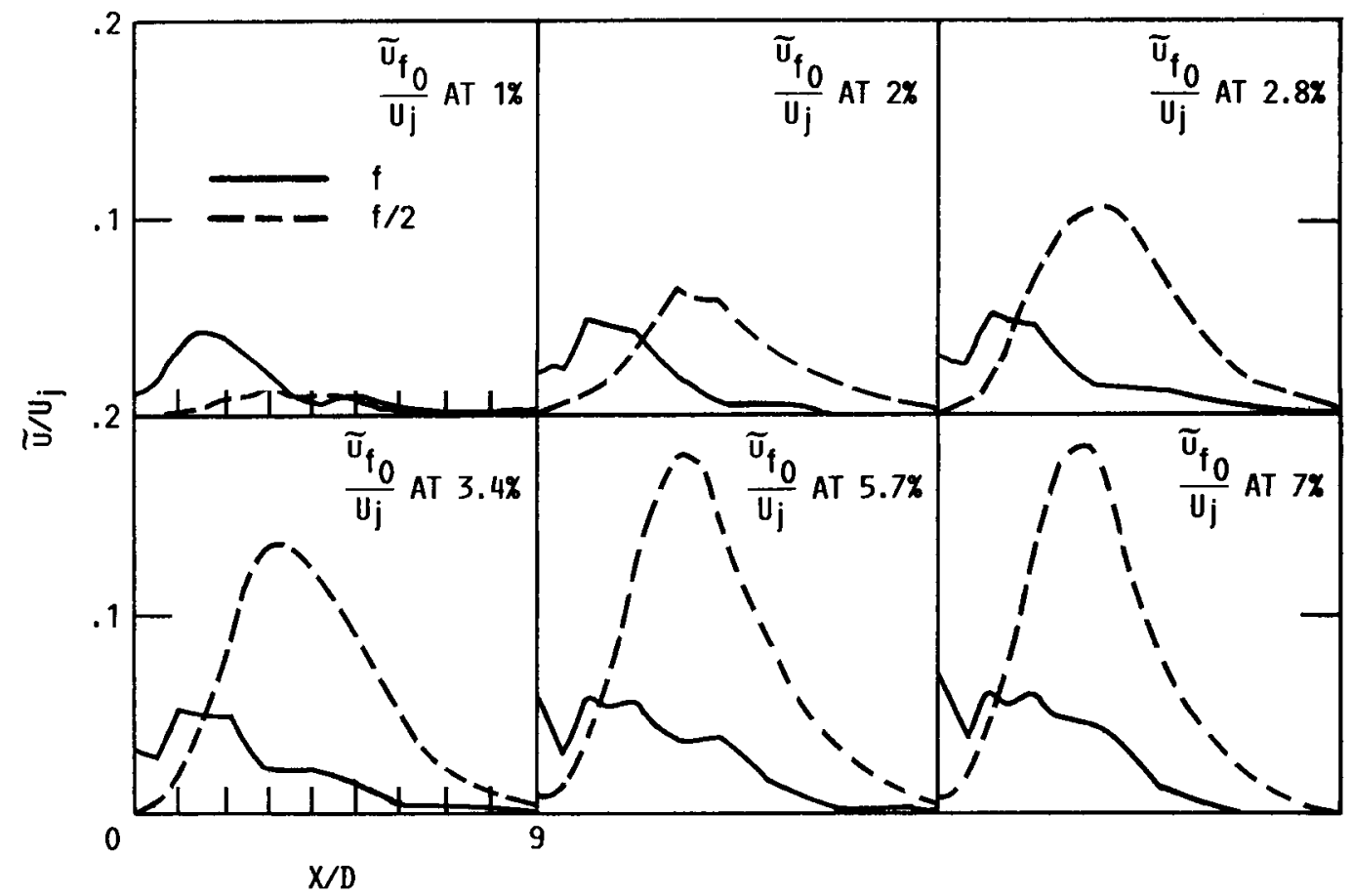

FIGURE 4(a). - AXIAL EVOLUTION OF PHASE-AVERAGED UNSTEADY VELOCITY COMPONENTS ON THE JET CENTERLINE FOR VARIOUS INITIAL LEVELS OF EXCITATON. $\quad(S t=0.2$, $0.4, \varphi_{0}=270^{\circ}, \widetilde{U}_{f_{0}} / \widetilde{U}_{f / 2}=15, M=0.45$.) 


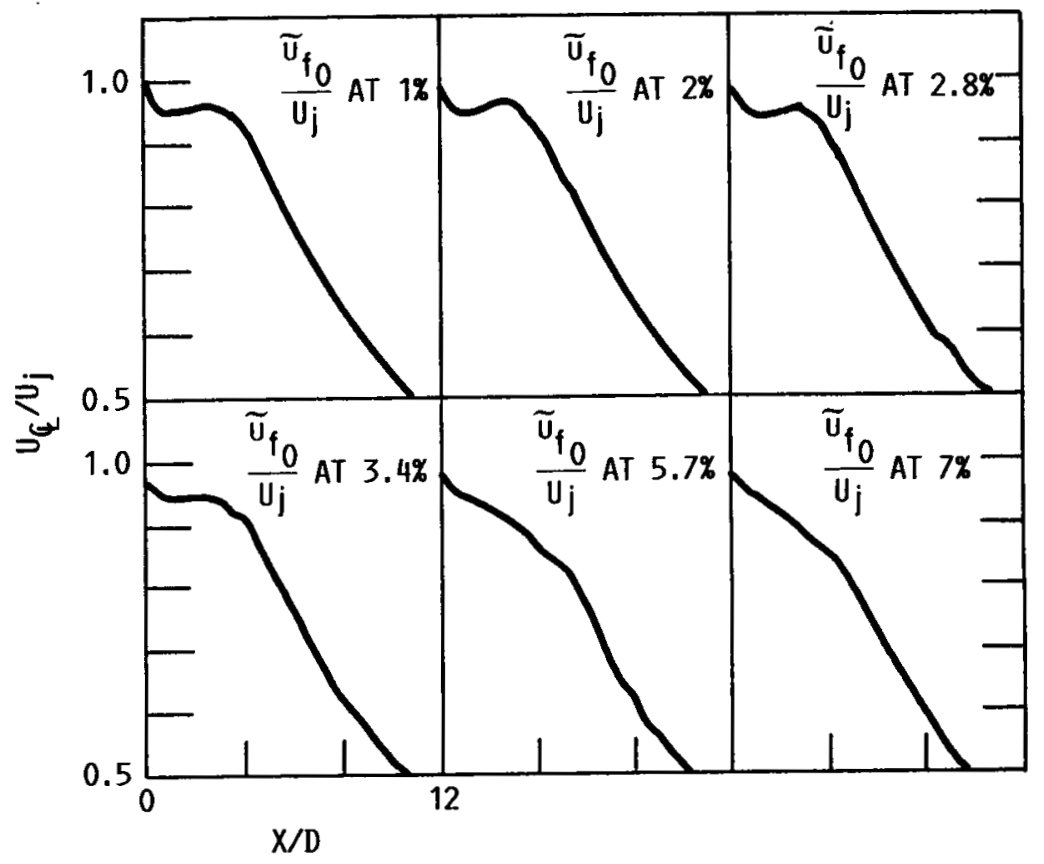

FIGURE 4(b). - EFFECT OF INCREASING INITIAL LEVELS OF EXCITATION ON THE JET CENTERLINE MEAN VELOCITY.

$\left(S t=0.2,0.4, \varphi_{0}=270^{\circ}, \widetilde{\mathrm{U}}_{\mathrm{f}} / \widetilde{\mathrm{U}}_{\mathrm{f} / 2_{0}}=15, M=0.45.\right)$ 


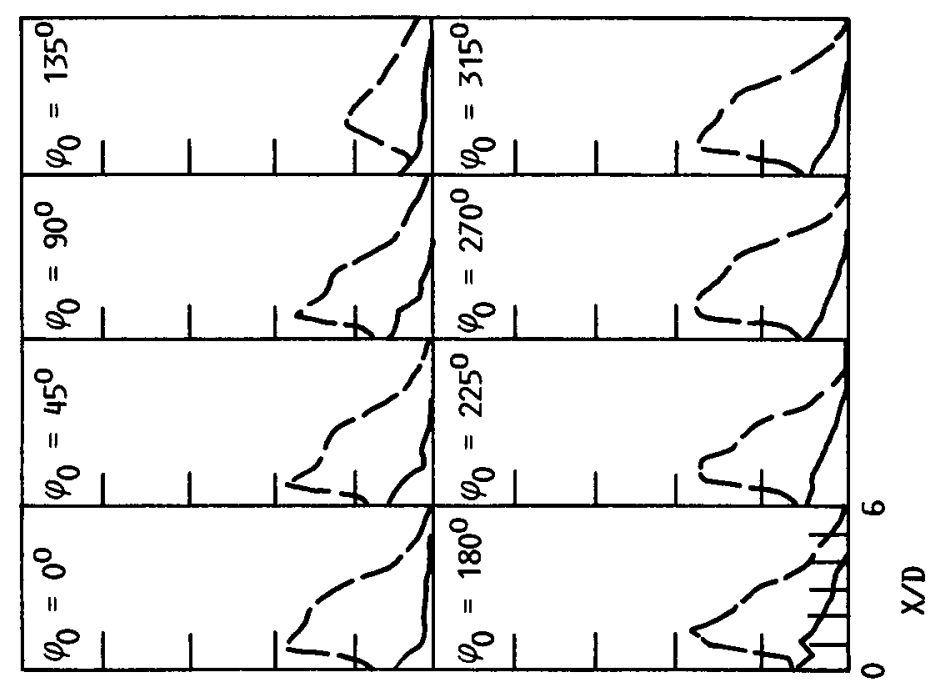

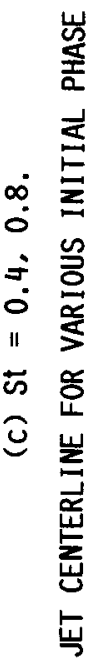

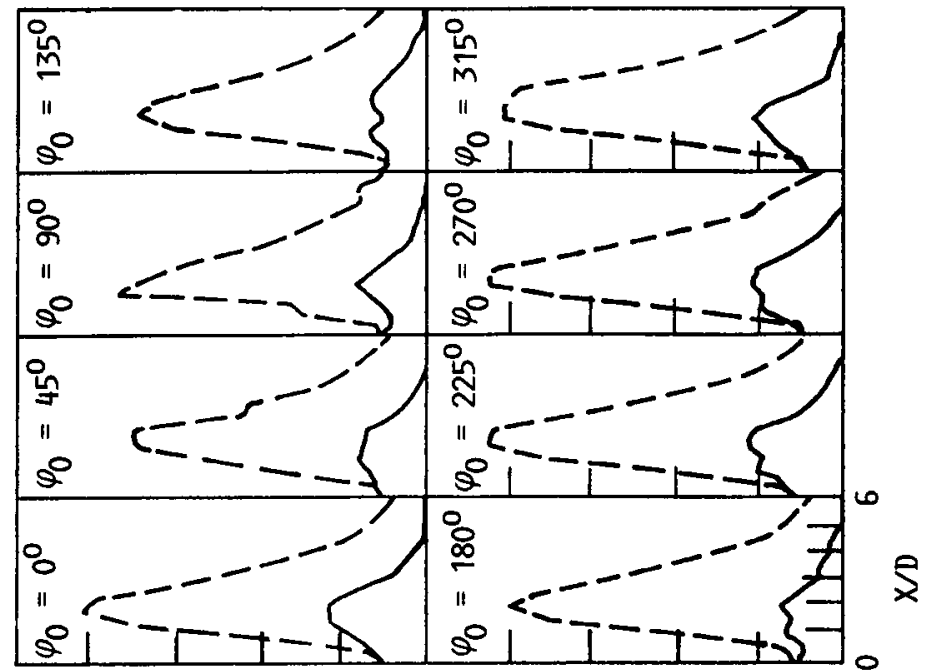

崖

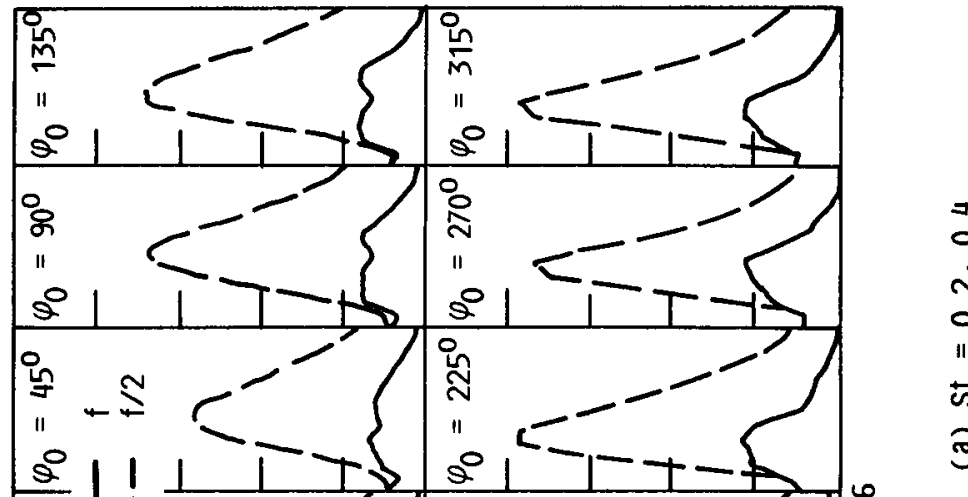

帘

ஸ்

" $\bar{\delta}$

क

อ

客

$\underset{x}{2}$

苞

送

文容崖

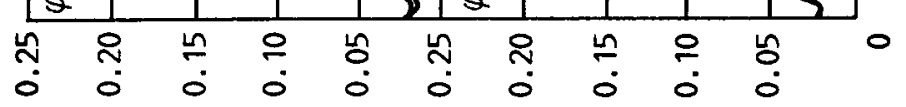

!n/n 


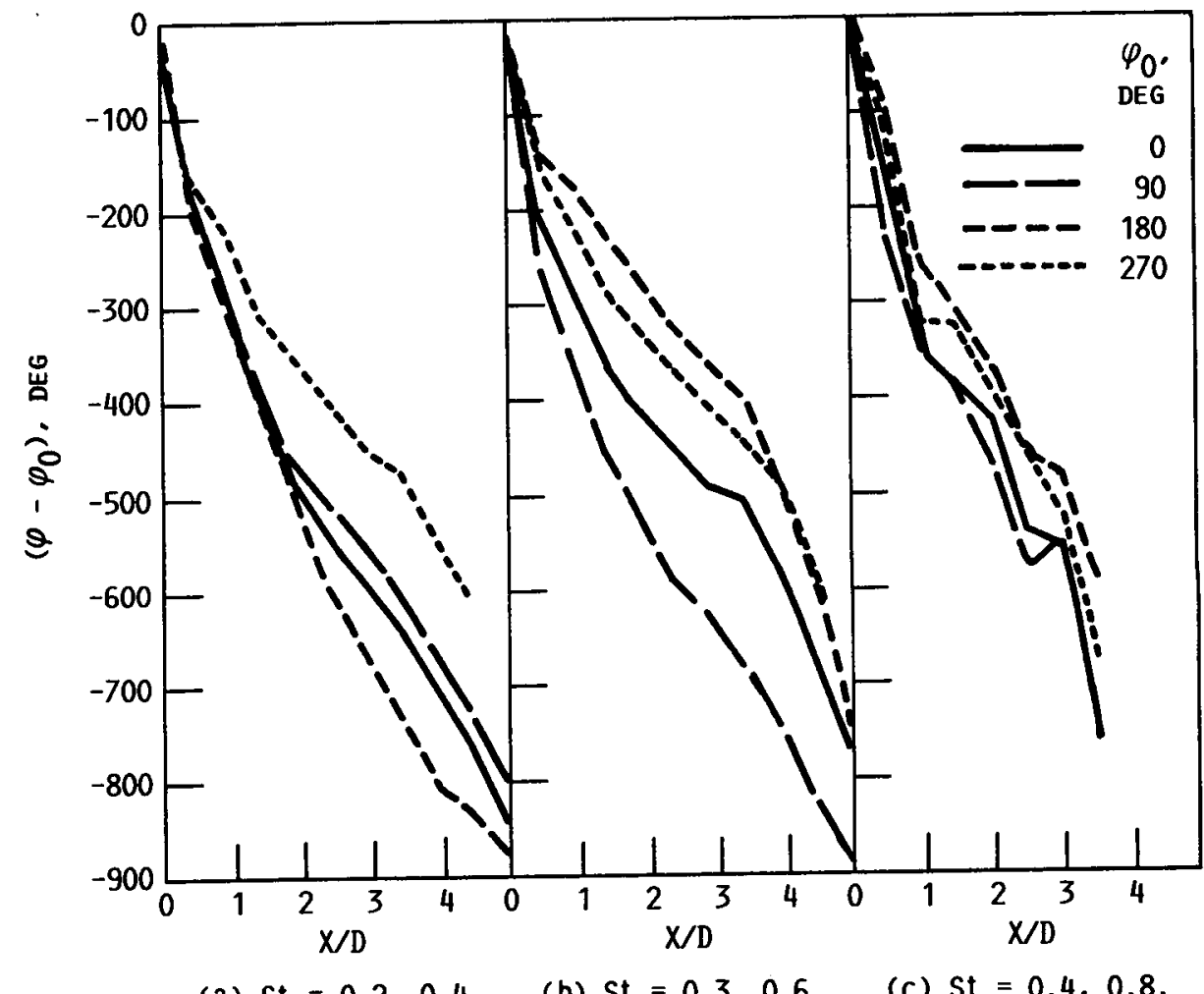

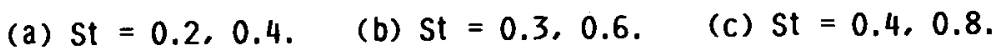

FIGURE 6. - EVOLUTION OF PHASE DIFFERENCE ON THE JET CENTERLINE, FOR VARIOUS INITIAL PHASE DIFFERENCES AND STROUHAL NUMBER PAIRS. $\quad\left(\widetilde{U}_{f_{0}}=\widetilde{u}_{f / 2}=0.003 U_{j}, M=0.2\right.$. $)$ 


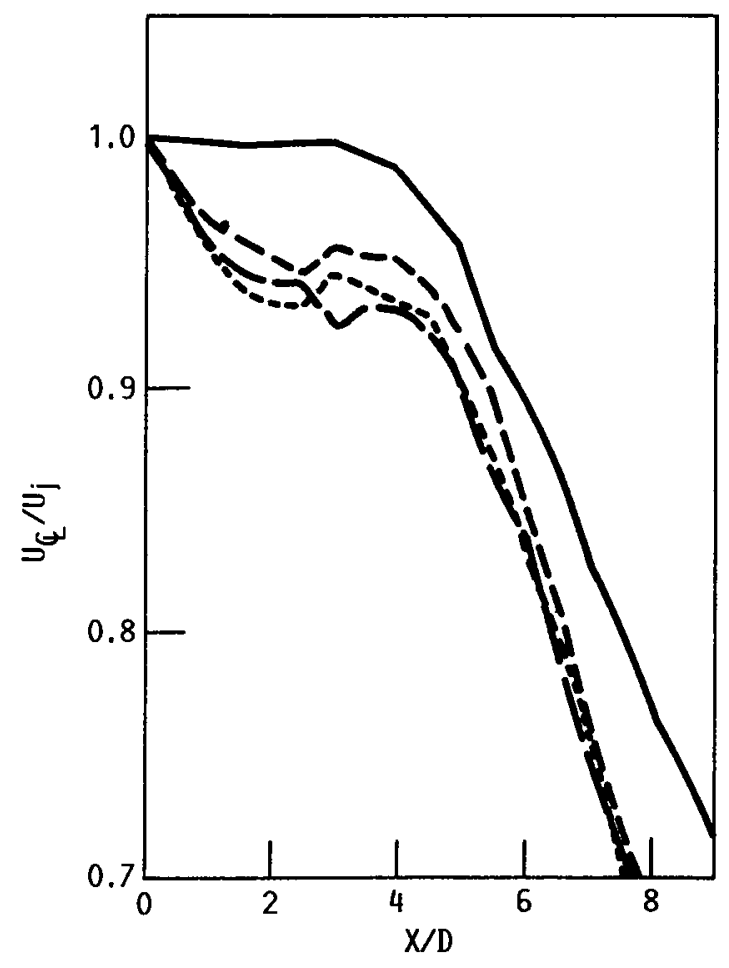

(a) AXIAL VARIATION OF CENTERLINE MEAN VELOCITY.

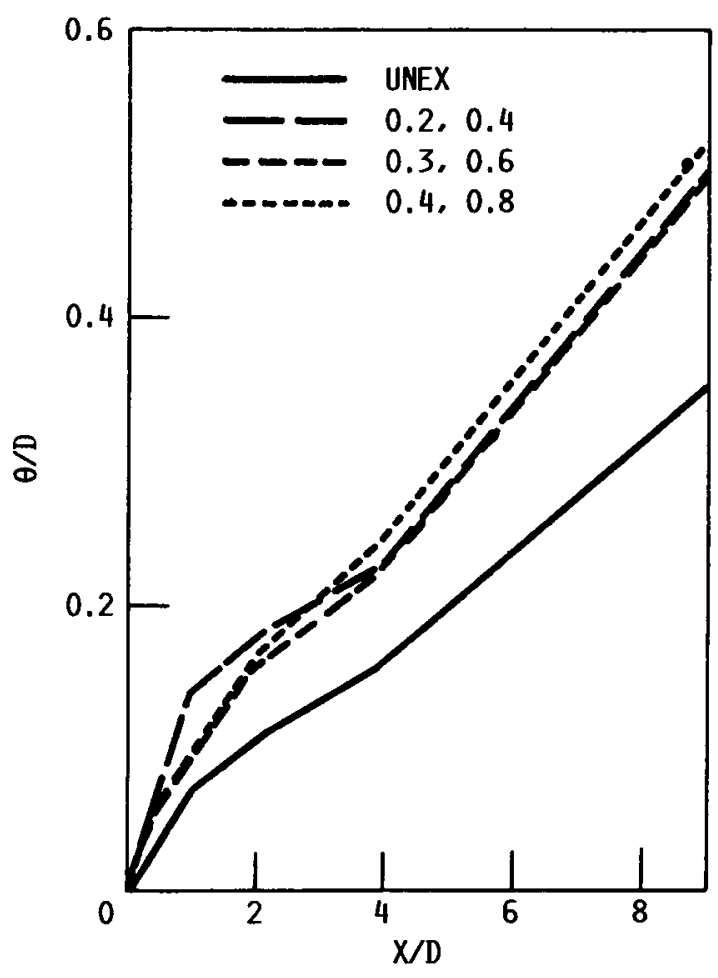

(b) AXIAL VARIATION OF MOMENTUM THICKNESS.

FIGURE 7. - TWO-FREQUENCY EXCITATION INFLUENCE ON JET CENTERLINE VELOCITY AND MOMENTUM THICKNESS FOR VARIOUS STROUHAL NUMBER PAIRS. $\left(\widetilde{U}_{f_{0}}=\widetilde{u}_{f / 2}=0.03 \mathbf{u}_{j} \cdot M=0.2\right.$. $\varphi_{0}=270^{\circ}$.) 

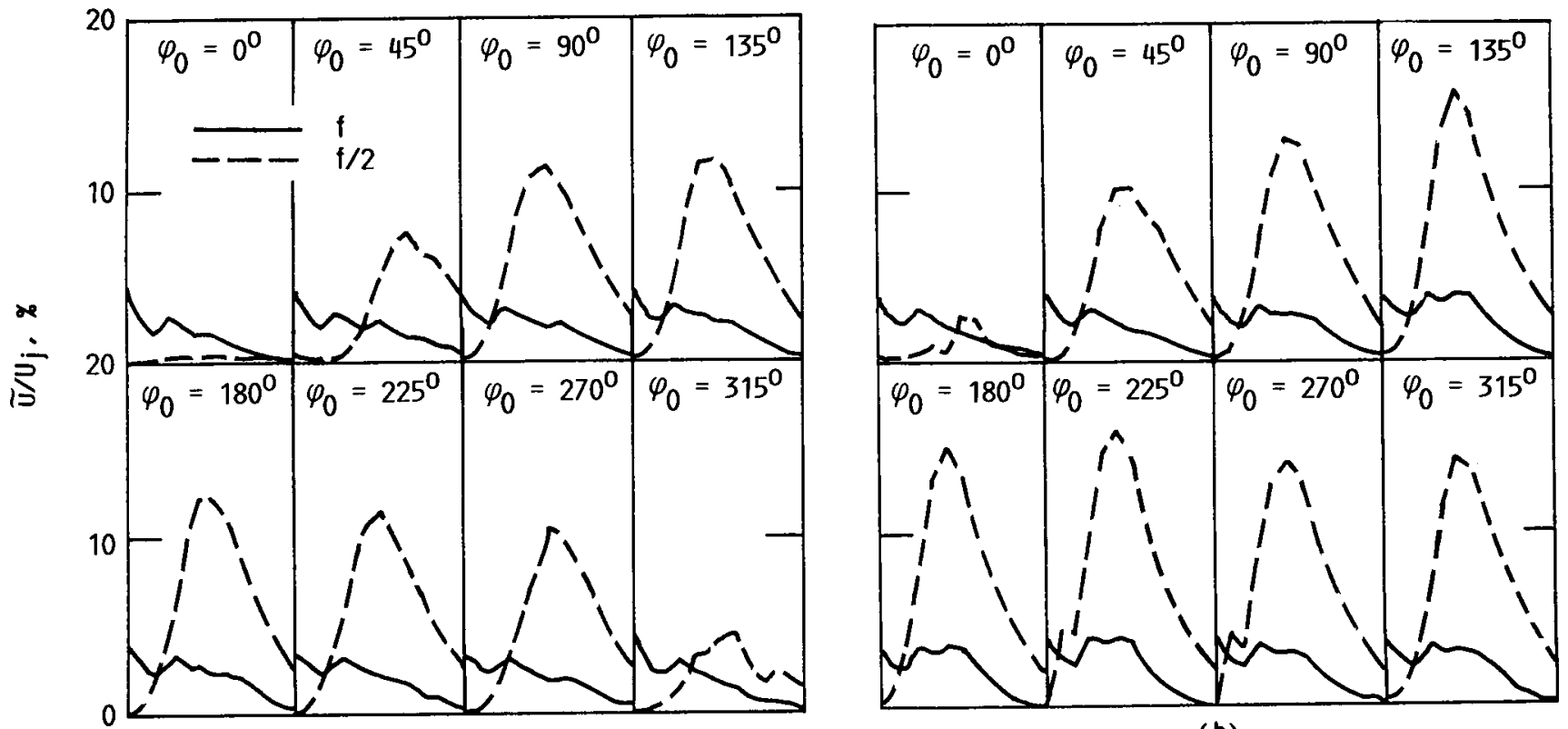

(a)

(b)
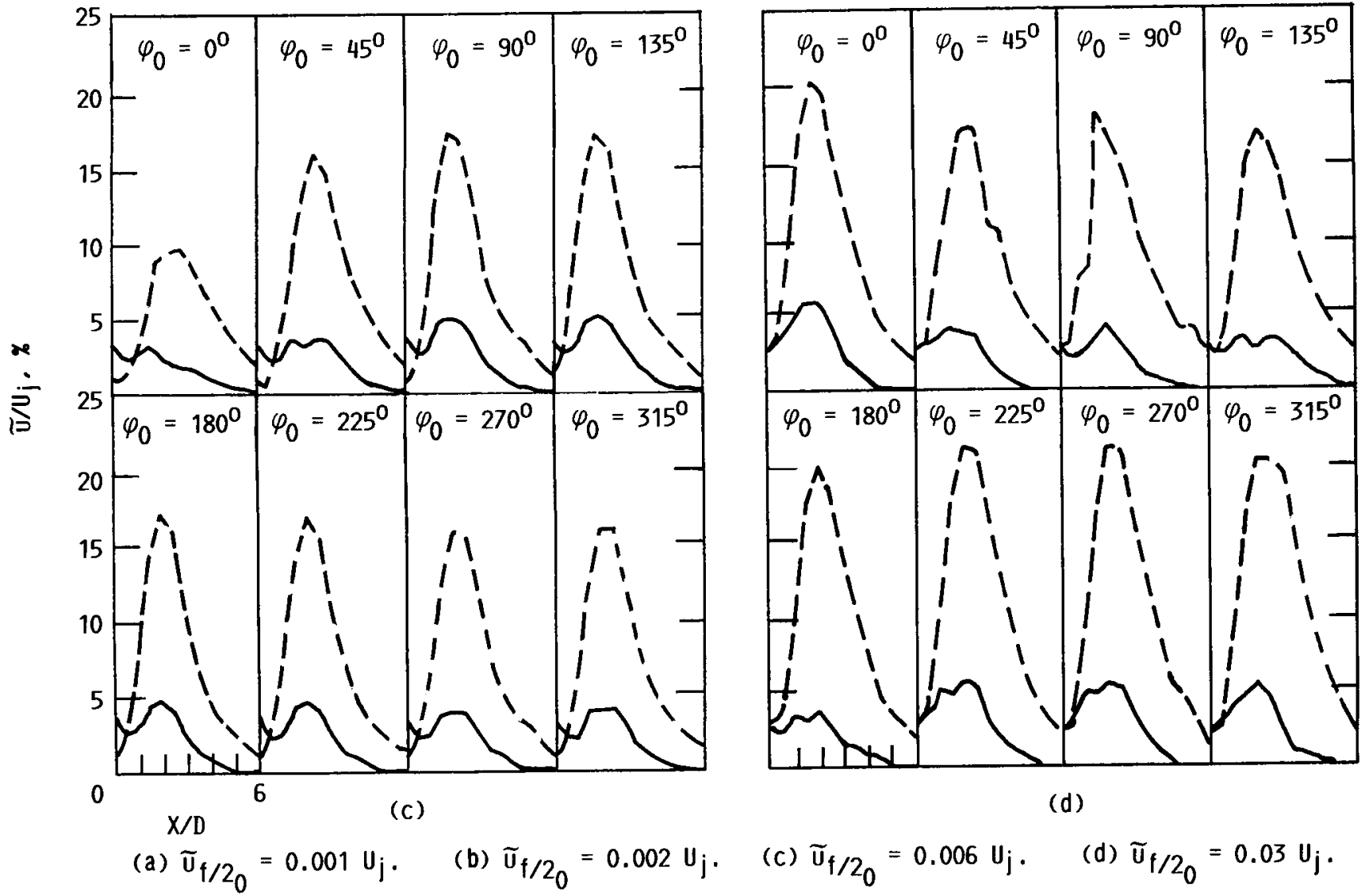

(d)

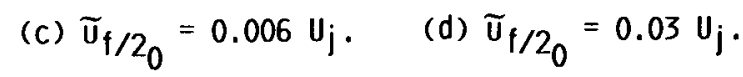

FIGURE 8. - AXIAL EVOLUTION OF PHASE-AVERAGED UNSTEADY VELOCITY COMPONENTS ON THE JET CENTERLINE FOR VARIOUS INITIAL PHASE DIFFERENCES AND SUBHARMONIC FORCING LEVELS. $(S t=0.3,0.6$, $\left.\widetilde{u}_{f_{0}}=0.03 U_{j}, M=0.2.\right)$ 


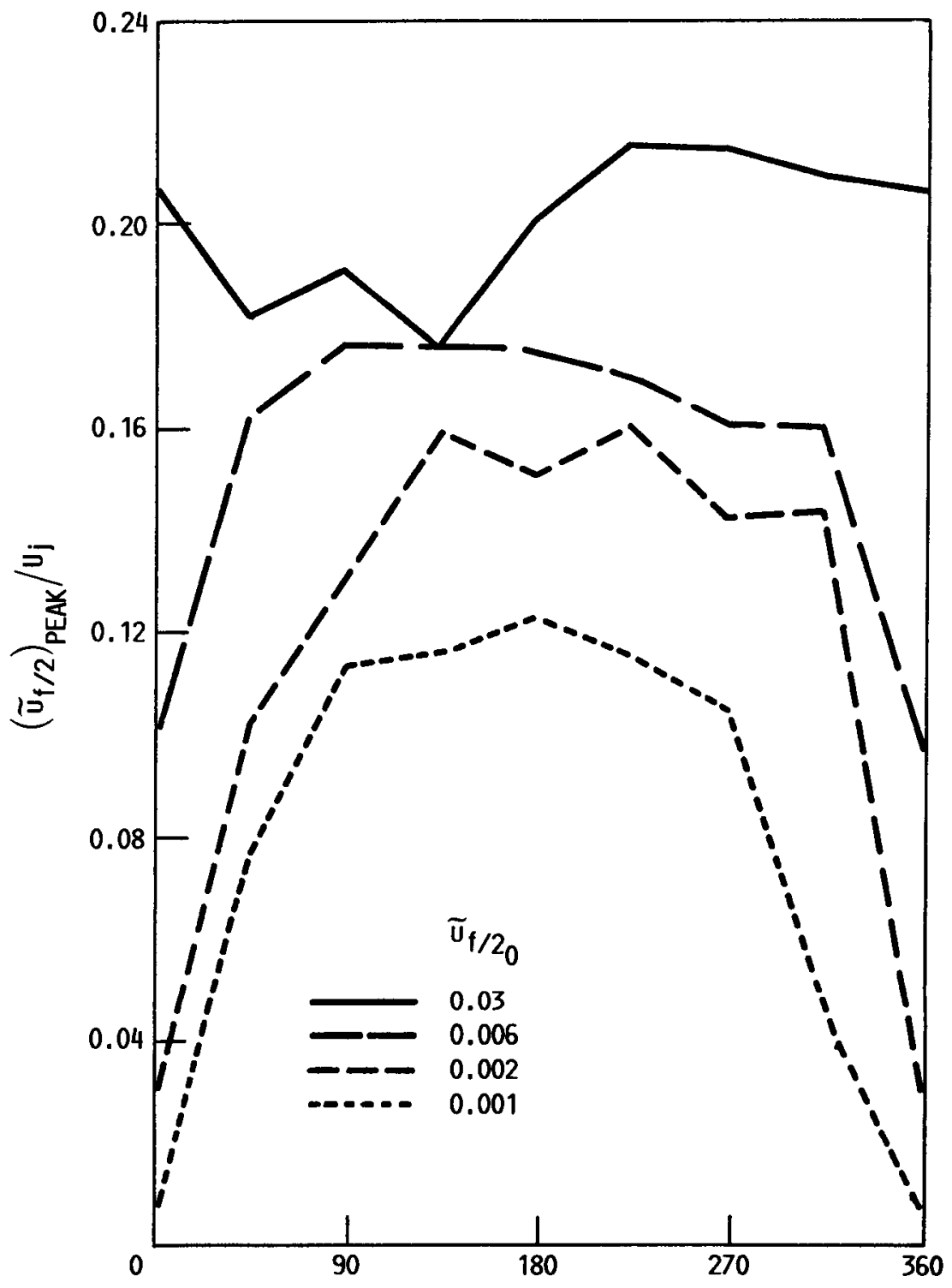

FIGURE 9. - SUBHARMONIC PEAK VARIATION WITH INITIAL PHASE DIFFERENCE FOR VARIOUS INITIAL SUBHARMONIC FORCING LEVELS. $\left(S t=0.3,0.6, \widetilde{v}_{f_{0}}=0.03 \mathrm{U}_{j}, M=0.2\right.$.) 


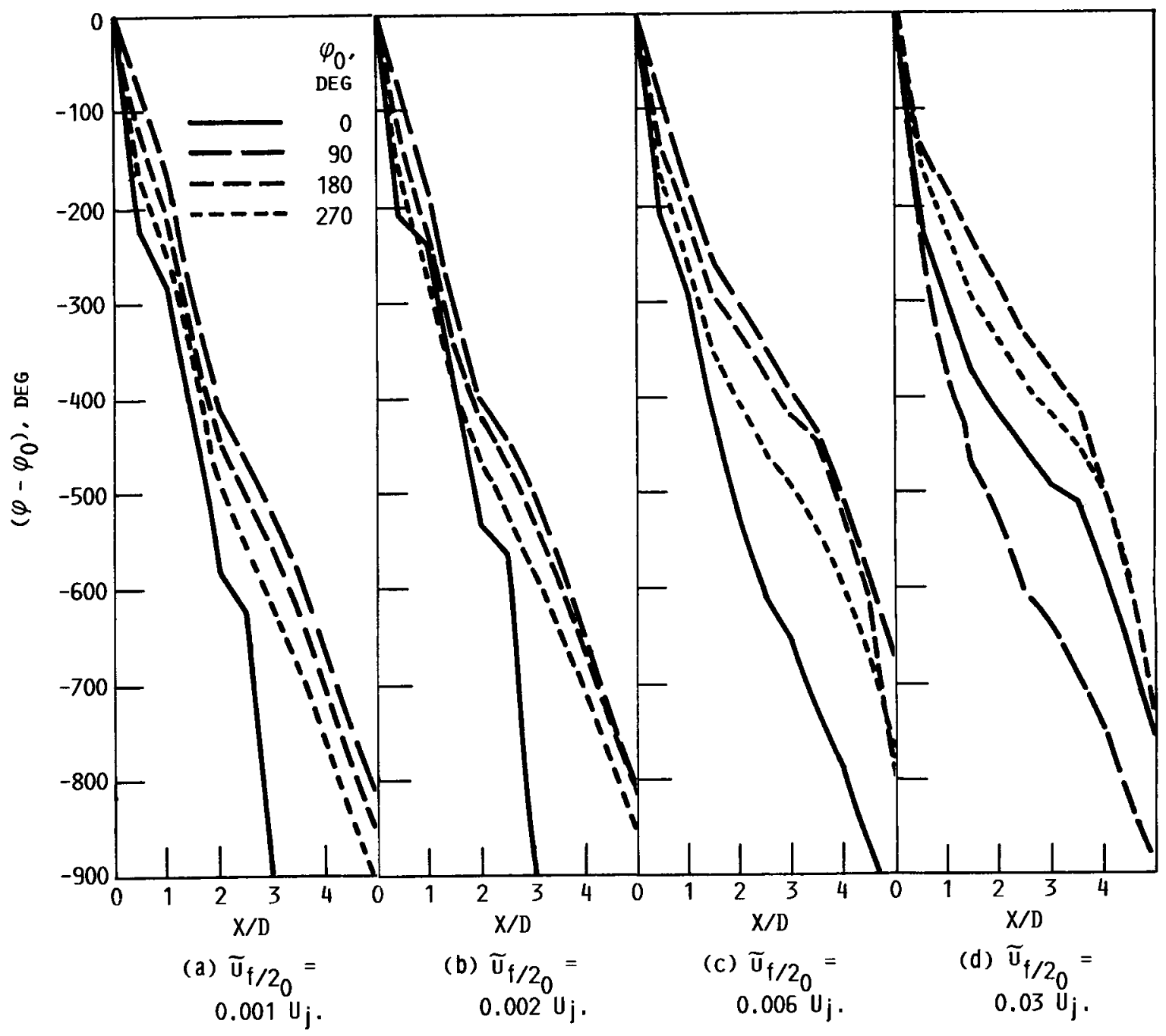

FIGURE 10. - EVOLUTION OF PHASE DIFFERENCE ON THE JET CENTERLINE, FOR VARIOUS INITIAL PHASE DIFFERENCES AND SUBHARMONIC FORCING LEVELS. $\left(S t=0.3,0.6, \widetilde{v}_{\mathfrak{f}_{0}}=0.03 \mathrm{u}_{\mathrm{j}}\right.$. $M=0.2$. ) 

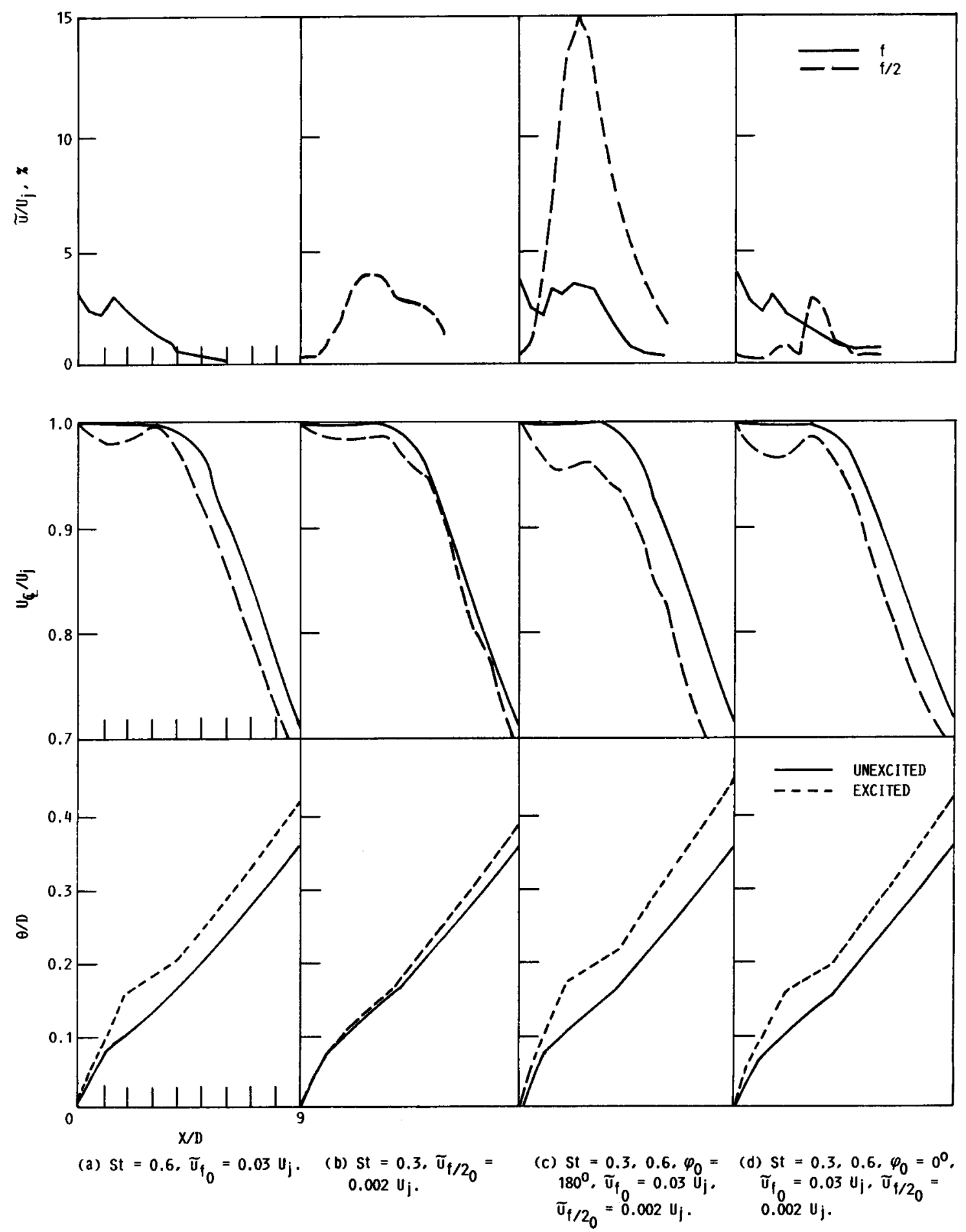

FigURE 11. - SUBhaRMONIC AUGMENTATION EFFECTS ON JET CENTERLIME VELOCITY AND MONENTLM THICKMESS. ( $m=0.2$.) 


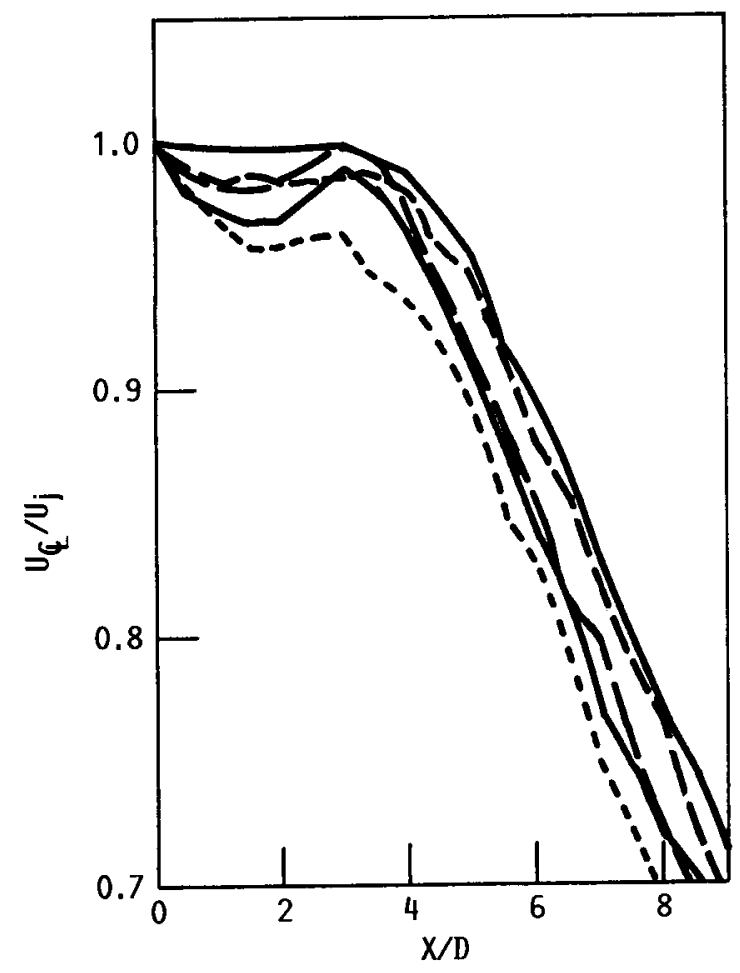

(a) AXIAL VARIATION OF CENTERLINE MEAN VELOCITY.

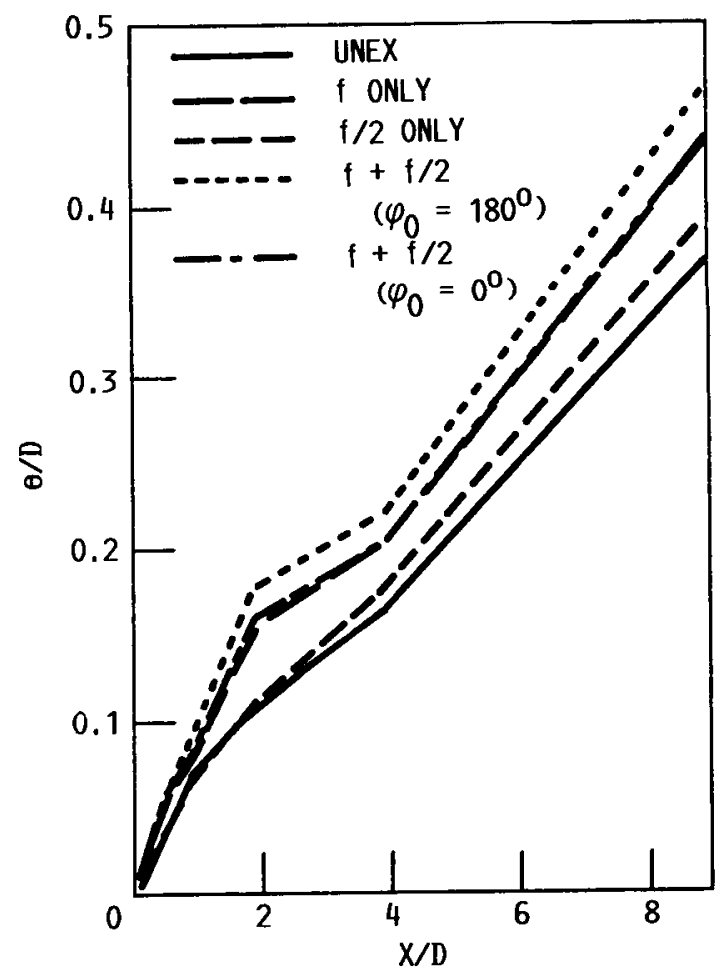

(b) AXIAL VARIATION OF MOMENTUM THICKNESS.

FIGURE 12. - JET CENTERLINE MEAN VELOCITY AND MOMENTUM THICKNESS, UNDER VARIOUS CONDITIONS OF EXCITATION. $\left(S t=0.3(f / 2)\right.$ AND $0.6(f), \widetilde{u}_{f_{0}}=0.03 u_{j}, \widetilde{u}_{f / 2}=0.002 U_{j}$, $M=0.2$.) 


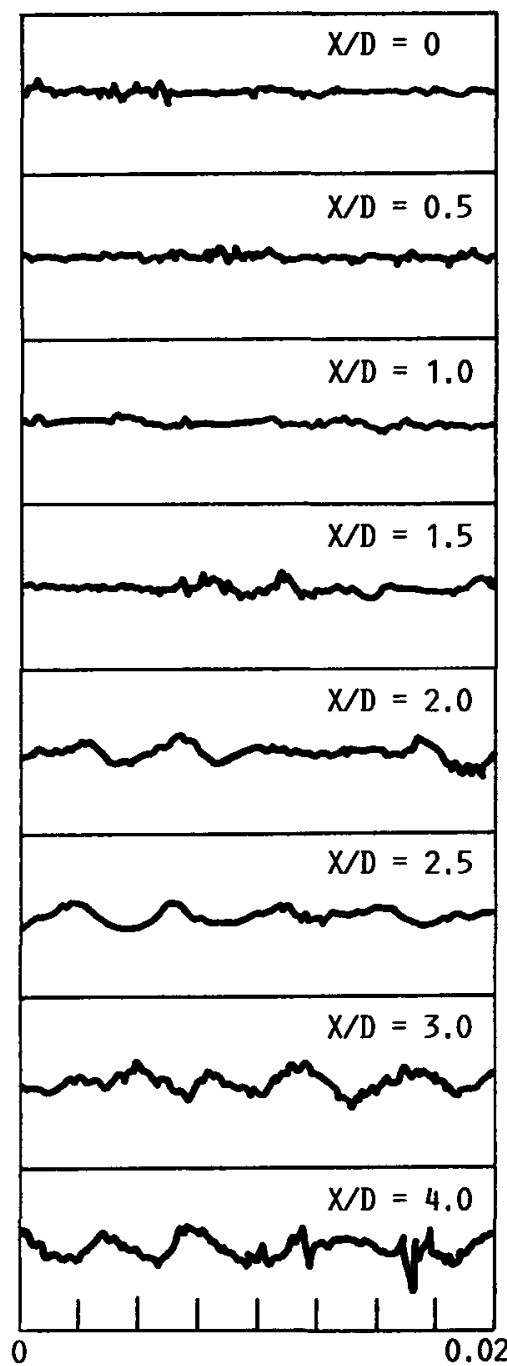

0.02

(a) UNEXCITED NATURAL JET.

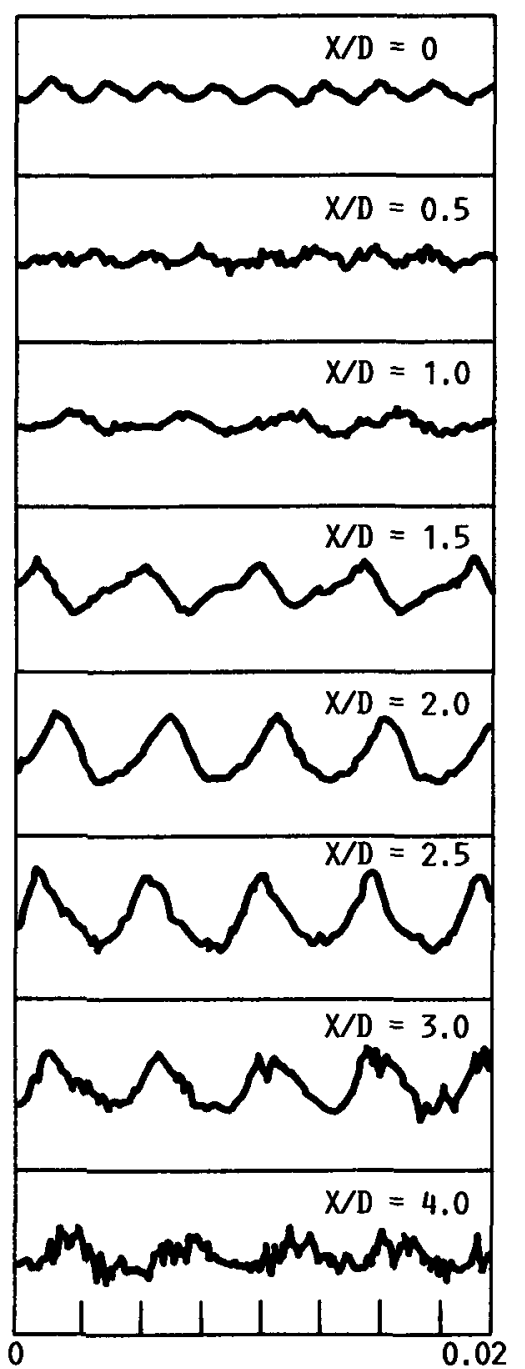

TIME, SEC

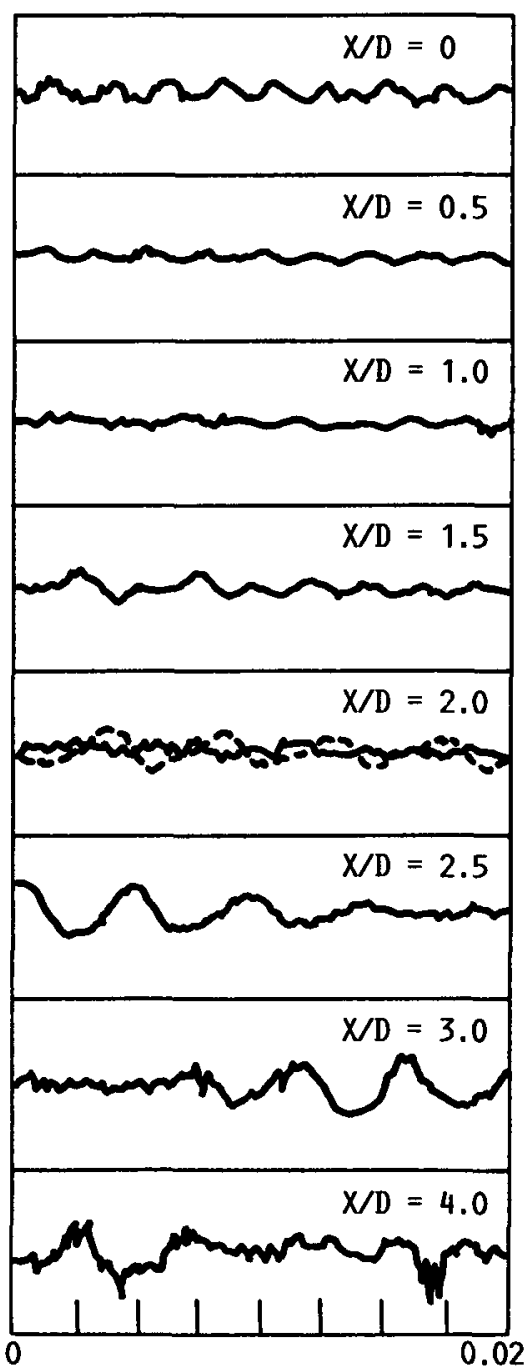

(c) EXCITED AT St $=0.3,0.6$, $\widetilde{u}_{f_{0}}=0.03 u_{j}, \widetilde{u}_{f / 2}=$ $0.002 \mathrm{U}_{\mathrm{j}} \cdot \varphi_{0}=0^{0}$.

FIGURE 13. - TIME TRACES OF THE VELOCITY SIGNAL AT VARIOUS AXIAL LOCATIONS ON THE JET CENTERL INE. 


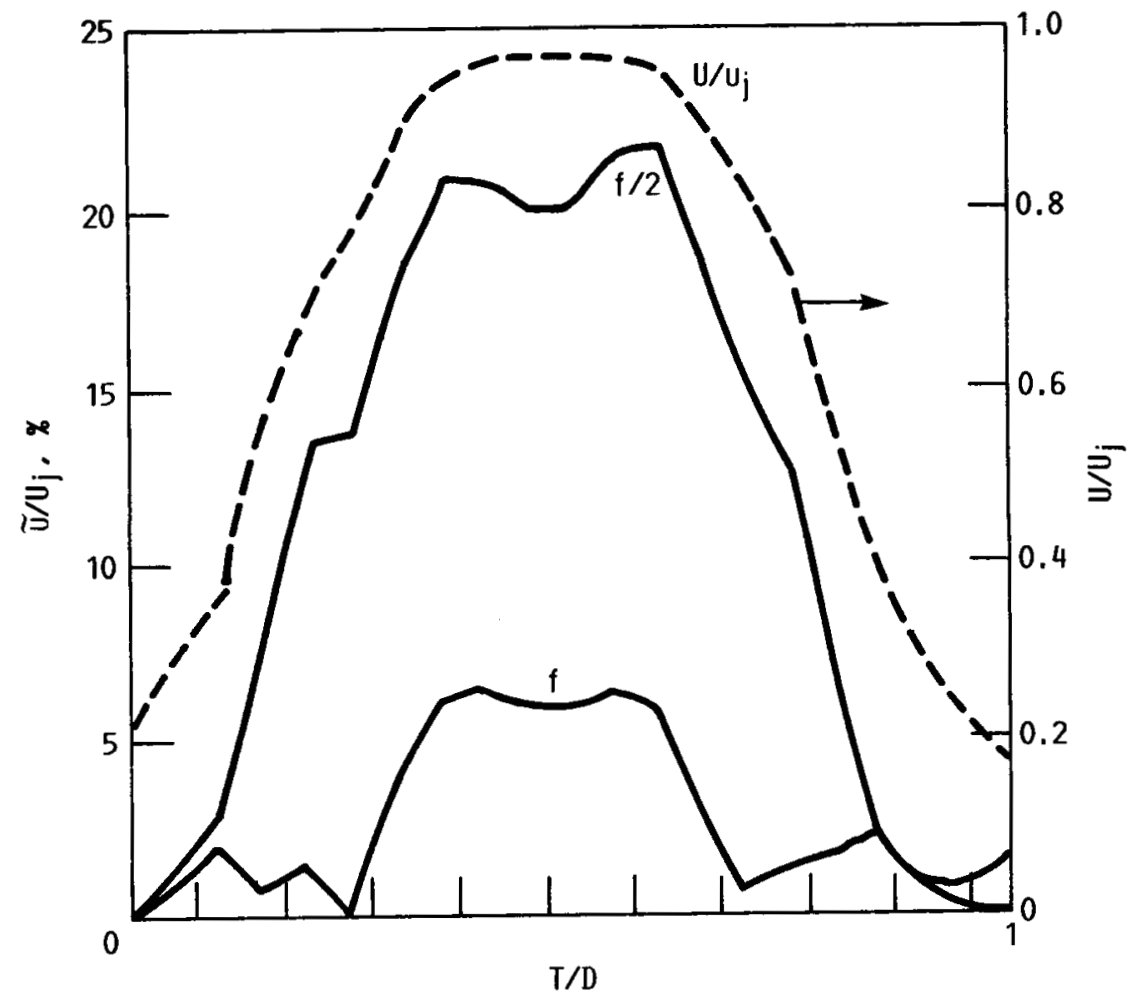

FIGURE 14. - RADIAL SHAPES OF FUNDAMENTAL AND SUBHARMONIC WAVES. $\left(S t=0.3,0.6, \widetilde{U}_{f_{0}}=0.03 \mathrm{u}_{j}\right.$, $\left.\widetilde{v}_{f / 2}=0.03 u_{j}, M=0.2, X / D=2, \varphi_{0}=0.\right)$ 


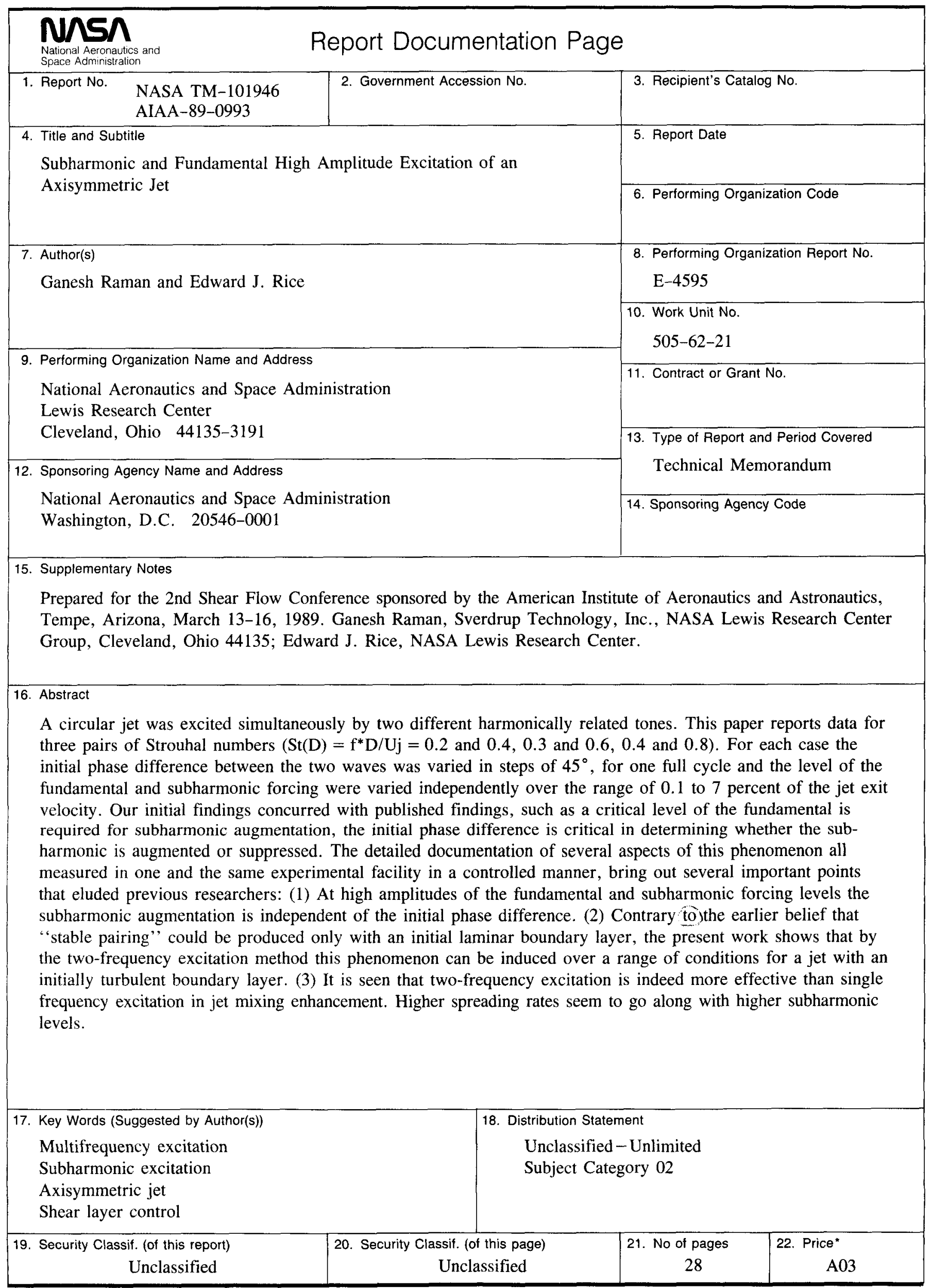

